\title{
Extent of Primary DNA Damage Measured by the Comet Assay in Health Professionals Exposed to Antineoplastic Drugs: A Systematic Review and Meta-Analysis
}

\author{
Vincenza Gianfredi ${ }^{1,2, *,+}$ (D) Daniele Nucci ${ }^{3,+}{ }^{\mathbb{C}}$, Cristina Fatigoni $^{1}{ }^{1}$, Tania Salvatori ${ }^{1}$, \\ Milena Villarini ${ }^{1}$ (I) and Massimo Moretti ${ }^{1, *}$ \\ 1 Department of Pharmaceutical Sciences (Unit of Public Health), University of Perugia, Via del Giochetto, \\ 06122 Perugia, Italy; cristina.fatigoni@unipg.it (C.F.); tania.salvatori90@gmail.com (T.S.); \\ milena.villarini@unipg.it (M.V.) \\ 2 Post Graduate School of Hygiene and Preventive Medicine, Department of Experimental Medicine, \\ University of Perugia, Piazzale Gambuli, 06122 Perugia, Italy \\ 3 Digestive Endoscopy Unit, IRCCS Istituto Oncologico Veneto, Via Gattamelata 64, 35128 Padova, Italy; \\ dnucci.prof@gmail.com \\ * Correspondence: vincenza.gianfredi@studenti.unipg.it (V.G.); massimo.moretti@unipg.it (M.M.); \\ Tel.: +39-07-5585-7488 (V.G.) \\ + Shared first authorship. These two Authors equally contribute to the manuscript.
}

Received: 12 December 2019; Accepted: 11 January 2020; Published: 14 January 2020

\begin{abstract}
Background: Antineoplastic drugs (ANDs) are a broad group of chemicals showing, at the same time, carcinogenic effects. The potential, albeit true, risk of side effects cannot be accepted, especially if resulting from occupational exposure. The aim of this study was to evaluate the association between occupational exposure to ANDs and the extent of primary DNA damage in health professionals. Methods: A systematic review and meta-analysis was conducted according to the Preferred Reporting Items for Systematic Reviews and Meta-Analyses guidelines. PubMed/Medline, Web of Science, and Scopus were used to perform the literature search. The databases were examined in July 2019. Sub-group, moderator, and cumulative analyses were conducted. The trim and fill method was used in the case of potential publication bias. Results: Twenty studies were included in the qualitative analysis, and 19 in quantitative evaluation. The pooled effect size was 1.27 [( $95 \%$ confidence interval $(\mathrm{CI})=0.66-1.88), p=0.000$ ] based on 1569 subjects. The moderator analysis by duration of exposure showed a positive association between duration of exposure and primary DNA damage. Conclusions: This systematic review clearly shows a significant association between occupational exposure to ANDs and the extent of primary DNA damage in health professionals. Considering these results, health professionals should be warned against this potential occupational risk.
\end{abstract}

Keywords: comet assay; single-cell gel-electrophoresis; antineoplastic drugs; occupational exposure; health personnel

\section{Introduction}

Antineoplastic drugs (ANDs) are a broad group of chemicals, mainly used in chemotherapy; their anti-proliferative effect was accidentally discovered during the First and Second World Wars, when bone marrow and lymph nodes depletion was registered in soldiers exposed to sulfur mustard [1,2]. However, the anticancer applicability of sulfur mustard was discovered in the late 1940s, and it was first used to treat non-Hodgkin's lymphoma and leukaemia [3,4]. ANDs can be classified in several 
modalities, such as, for instance, considering their mechanism of action, chemical structure, origins, or phase-specific toxicity; however, regardless of the classification used, ANDs show, along with their anti-proliferative properties, a certain amount of carcinogenic effects. ANDs are considered "possibly (group 2B), probably (group 2A) and carcinogenic to humans" (group 1) by the International Agency of Research on Cancer (IARC, Lyon, France) [5].

The potential risk of side effects, including secondary cancers, is bearable and accepted by the scientific community and by most cancer patients, as ANDs are able to increase survival. However, the risk cannot be tolerated, especially if resulting from occupational exposure. To date, several studies have shown the potential association between occupational exposure to ANDs and side effects on health professionals, both considering genotoxic [6,7] and non-genotoxic effects (i.e., teratogenicity, infertility) [8-10]. Genotoxic effects have been mainly evaluated through molecular epidemiology studies, in which biomarkers have been used to assess the effect of exposure to ANDs [11]. Numerous genotoxic biomarkers have been described in the literature, most of which are used to identify early cytogenetic effects—such as, for instance, micronuclei, sister chromatid exchanges, and chromosome aberrations-or reversible DNA damage—such as DNA adducts and primary DNA damage [4]. The latter is considered as a biomarker of effective biological dose at the target level and can be assessed through the comet assay (also known as the single-cell gel-electrophoresis test). This procedure was first introduced by Östling \& Johanson in 1984 [12] and subsequently modified by Singh et al. in 1988 [13]. The standard alkaline procedure of the comet assay (i.e., lysis at $\mathrm{pH} 10$, unwinding, and electrophoresis at $\mathrm{pH}>13$ ) allows the detection of both single- and double-strand DNA breaks, apurinic/apyrimidinic sites (i.e., alkali labile lesions) that are expressed as frank strand breaks in the DNA under the alkaline conditions of the assay, as well as open sites formed during DNA repair incision [14]. The comet assay is relatively easy to perform, thanks to the low number of eukaryotic cells needed, and it is a high sensitivity test able to detect a break in the DNA strand every $10^{10}$ Daltons [15]. Considering these important advantages, the comet assay has been recently considered applicable for occupational exposure biomonitoring [16-18].

The first aim of this systematic review and meta-analysis was to identify all the published studies assessing the occupational exposure risk to ANDs for health professionals, measured by the comet assay. The second aim was to retrospectively analyze and synthesize the available evidence. The last study aim was to combine the collected data in order to perform a meta-analytic evaluation of the risk of DNA damage comparing health professionals occupationally exposed to ANDs and health professionals not exposed to ANDs.

\section{Materials and Methods}

The Preferred Reporting Items for Systematic Reviews and Meta-Analyses (PRISMA) guidelines were used in order to complete the following systematic review and meta-analysis [19]. The structured computer literature search was performed using the following electronic databases: PubMed/Medline, Web of Science, and Scopus. The search strategy was developed with a combination of pre-determined keywords, using both Medical Subject Headings (MeSH) and text words terms according to the type of database consulted. In order to identify a proper set of keywords, three components were taken into account: health professional exposure, antineoplastic drugs, and comet. The identified keywords were then combined using Boolean operators AND/OR. The search strategy is available upon request. The databases were examined in July 2019.

\subsection{Inclusion/Exclusion Criteria}

Two researchers independently screened titles and abstracts of the retrieved references, in order to assess compliance with inclusion/exclusion criteria. In order to be included in this review, the studies were required to report the results of primary research evaluating ANDs exposure in health care professionals (both women and men), as well as the risk of ANDs exposure measured with comet assay. Furthermore, we only included studies published in English and with full text available. 
Besides, only human studies, with original data (e.g., reviews, letters to the editor, and commentaries were excluded), focusing strictly on our outcome of interest expressed as mean \pm standard deviation (SD), were included. In order to perform a very comprehensive analysis, reducing the possibility of losing studies, no time filter was added. A more detailed explanation of inclusion/exclusion criteria, according to a population, intervention, comparison, outcomes, and study design (PICOS) [20], is reported in Table 1 . The PICOS criteria were extended with time and language filters, as suggested by the Cochrane Collaboration [21].

Table 1. Detailed inclusion/exclusion criteria, according to a population, intervention, comparison, outcomes, and study design (PICOS) statement extended with a language and time filter.

\begin{tabular}{cc}
\hline Search Strategy & Details \\
\hline P: health professionals occupationally exposed to antineoplastic drugs (ANDs) \\
(female and male)
\end{tabular}

\subsection{Data Extraction}

Full text was downloaded only for eligible articles. Data extraction was conducted only for articles included according to the previously mentioned criteria. Two researchers independently performed data extraction using a pre-defined and pre-piloted spreadsheet elaborated in Microsoft Excel ${ }^{\circledR}$ for Windows. Any potential disagreement was solved through discussion between the two researchers or, if disagreement persisted, by consulting a third researcher. Data extracted from the original studies were both quantitative and qualitative. Qualitative data recorded included the following: name of first author, year of publication, country where the study was conducted, and preventive measures adopted. Moreover, characteristics of the subjects were also recorded (e.g., age, gender, and job task). The quantitative data extracted were as follows: sample size, ward of the participants, duration of the exposure (expressed in years), and outcome (expressed as mean). In the case of missing details, the corresponding author of the original manuscript was contacted by e-mail. A crosscheck of the reference lists was performed in order to identify further potentially related articles. References were managed using the citation manager software EndNote.

\subsection{Quality Evaluation}

Two researchers independently performed the quality evaluation of the included articles. A validated tool comprising nine items assessing several methodological characteristics of primary studies (i.e., number of exposed and controls, matching for multiple risk factors, and number of cells scored per subject) was used for evaluation. For each item, the score could range from 1 to 3 , while the total score could range from 9 to 27 points [7].

\subsection{Statistical Analysis}

The effect size (ES) was calculated based on the mean, standard deviation (SD), and sample size provided per each study. It was estimated by Cohen's $d$, reported with its $95 \%$ confidence interval (CI). Cohen's d is the preferred method of ES calculation, and it is calculated as a difference between two means, divided by the variability among the sample. In this way, it is possible to compare results expressed with different units of measures [22]. The comparison was performed between subjects 
exposed to the antineoplastic drugs and subjects without any type of exposure. In order to reduce the heterogeneity, a random-effect model was applied to conduct the meta-analysis. The heterogeneity among the included studies was evaluated through $\mathrm{Chi}^{2}$ and $\mathrm{I}^{2}$ tests. An $\mathrm{I}^{2}$ value below $25 \%$ means no heterogeneity, a value ranging between $25 \%$ and $50 \%$ is considered as low, while values between $50 \%$ and $75 \%$ are considered as moderate, lastly a value $>75 \%$ means high heterogeneity. Potential publication bias was evaluated through visual examination of the funnel plot and by the Egger's regression asymmetry test. The funnel plot graphically represents the distribution of the retrieved studies considering ES and standard error. It is used for visually detecting potential publication bias based on the symmetry of the plot. However, as the visual examination could be subjective, the Egger's regression asymmetry test was used as well, being able to provide an objective measure for publication bias. For the Egger's regression asymmetry test, the statistical significance was set at $p<0.10$ [23]. If publication bias was detected, a trim and fill method was used in order to adjust for publication bias [24]. The trim and fill method is a statistical approach aimed to estimate potential missing studies, causing the asymmetry of the funnel plot. This method assumes that the studies with the most extreme ES have to be suppressed, adjusting the overall effect estimate [25]. To perform the meta-analysis, the software Prometa3 ${ }^{\circledR}$ (Internovi, Italy) was used.

\subsection{Sub-Group and Sensitivity Analysis}

In order to reduce the heterogeneity, two sensitivity analyses were conducted, considering the following: (i) only studies with higher quality score (QS $\geq 16$ ), and (ii) combining studies with the same unit of measure. The subgroups analyses are commonly performed because the effect of a measurement might vary among subgroups of subjects, defined by individual characteristics. In order to consolidate the validity of the results, several sub-group analyses were developed, taking into consideration the following: gender (male and female separately), protective equipment used (nothings or gloves and masks versus at least gloves, masks, and air-flow cabinet), and work task (excluding studies that considered nurses not exposed to ANDs as controls).

\subsection{Cumulative Analysis}

A cumulative analysis is a sequence of meta-analyses, aiming to estimate the changes of the ES starting with a single study and adding the other studies one at a time. The cumulative analysis accumulates the results from the first to the latest study, and each successive result includes a synthesis of all previous studies. This analysis expresses the potential consistency of the results [26]. We performed the cumulative analysis both chronologically (adding each study according to the year of publication) and considering the sample size (from smallest to biggest).

\subsection{Moderator Analysis}

To explore potential moderators of these observed effects, owing to a third variable, we examined the variation in ES associated with the continent where the studies were conducted (categorized in Europe, America, and Asia), and year of publication, categorized into three groups (the first group consisted of studies until 2004, the second between 2005 and 2010, and the third after 2010 until today). These three groups were chosen considering that main guidelines on ANDs manipulation-aimed at reducing the occupational risk-were published in 2004 [27], in 2006 [28], and in 2009 [29]. Moreover, a meta-regression analysis was conducted in order to examine the impact of the duration of occupational exposure (expressed in years), and exposed group's mean age (expressed in years) on ES.

\section{Results}

\subsection{Literature Search}

A total of 373 articles were identified-69 in Web of Science database, 149 in PubMed/Medline, and 155 in Scopus database. A total of 85 documents were immediately excluded because of duplicates. 
After the preliminary screening by title and abstract, 267 documents were excluded because they were either reviews $(n=53)$, conference papers, or letters to the editor $(n=13)$, or because of unrelated topics $(n=172)$ or different languages $(n=29)$. Out of the 29 articles excluded because of language restriction, we identified articles written in Chinese $(n=6)$, Italian $(n=4)$, French $(n=4)$, Hungarian $(n=3)$, Serbian $(n=2)$, Czech $(n=2)$, Finnish $(n=1)$, German $(n=1)$, Japanese $(n=1)$, Korean $(n=1)$, Polish $(n=1)$, Portuguese $(n=1)$, Russian $(n=1)$, and Spanish $(n=1)$. Overall, 21 articles were eligible, but one reference was excluded because it was an in vitro study. Figure 1 shows the flow diagram, reporting the selection process. At the end of the screening process, 20 articles were included in the qualitative analysis [30-49], and 19 in the quantitative analysis. One study was removed from the quantitative analysis because, even if the authors had been contacted in order to collect further information, data were not available [49]. Moreover, because four papers reported the results both aggregated and divided by gender, we included the latter data in the sub-group analysis by gender $[33,35,40,47]$. In order to perform a robust sub-group analysis, when possible, data divided by gender were calculated from the original studies [34]. At the same time, the study conducted by Ursini et al., 2006 [46], showed the results divided by work task, and Kopjar et al., 2001 [37] showed the results divided by protective equipment used; for these reasons, these data were included in the sub-group analysis.

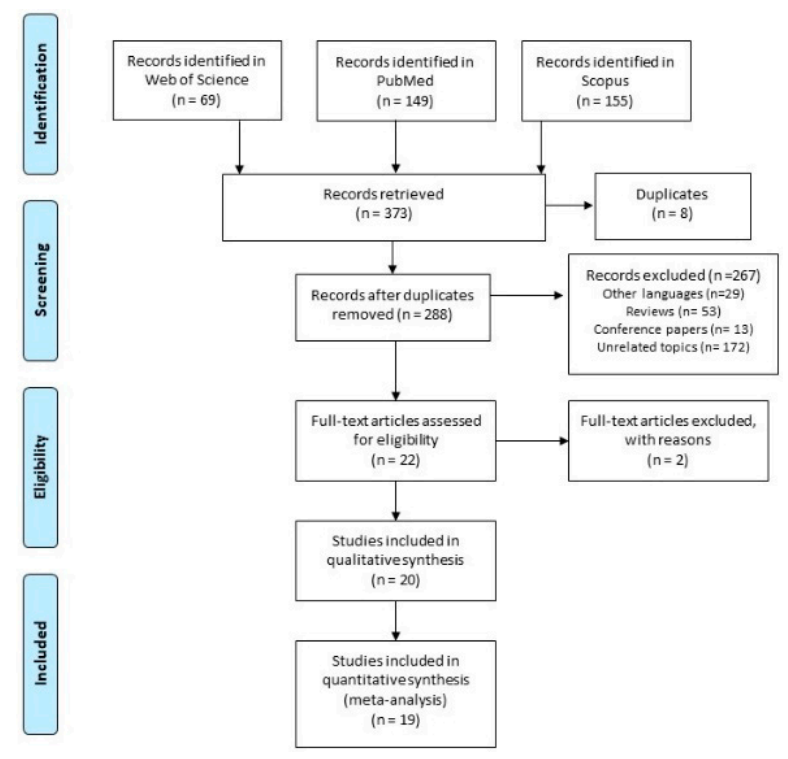

Figure 1. Flow diagram of the selection process.

\subsection{Characteristics of the Included Studies}

Most of the included studies were conducted in Western countries, in particular, 10 were conducted in Europe [Italy $(n=5)$, Portugal $(n=2)$, Croatia $(n=2)$, and Austria $(n=1)$ ] and 3 in the Americas [United States $(n=1)$ and Brazil $(n=2)]$. The remaining studies were conducted in Asia, particularly China $(n=2)$, Turkey $(n=2)$, Japan $(n=2)$, and India $(n=1)$. The majority of the studies performed the comet assay using lymphocyte cells, while two studies incorporated the results of the comet assay of buccal cells [31,46]. Most of the studies were published before $2010(n=17)$; the oldest study was published in 1999 [45], while the newest retrieved study was published in 2015 [39]. All the included studies except one [49] performed a cross-sectional analysis considering professionals exposed to ANDs compared to others professionals not occupationally exposed to ANDs. Only one study was followed by a follow-up [49]; in this study, the authors estimated the correlation between ANDs' exposure and comet assay by trend analysis, considering the individual risk with regard to the years of exposure. Concerning the quality evaluation, the score ranged between 11 and 19, with a mean of 16 (Table 2). 
Table 2. Descriptive characteristics of the included studies.

\begin{tabular}{|c|c|c|c|c|c|c|c|c|c|c|}
\hline Author, Year & Country & Gender & $\begin{array}{c}\text { Mean Age } \pm \text { SD } \\
\text { (Years) }\end{array}$ & $n(\mathrm{E} / \mathrm{C}) \ddagger$ & Work Task & $\begin{array}{l}\text { Mean Exposure } \pm \\
\text { SD (Years) }\end{array}$ & Protective Equipment & Cells & $\begin{array}{l}\text { Comet Test } \\
\text { Mean } \pm \text { SD }\end{array}$ & $\mathrm{QS} / 27$ \\
\hline Bruschini A et al., 2013 & Italy & $\mathrm{F}$ & $\begin{array}{l}E \ddagger=39.0 \pm 8.0 \\
C \neq=40.0 \pm 9.0\end{array}$ & $63 / 74$ & $\begin{array}{l}\text { Hospital nurses } \\
\text { Hospital nurses }\end{array}$ & $9.2 \pm 7.2$ & n.a. & lymphocytes & $\begin{array}{l}0.95 \pm 003 \\
0.99 \pm 0.03\end{array}$ & 15 \\
\hline Cavallo D. et al., 2009 (a) & Italy & $\mathrm{M}+\mathrm{F}$ & $\begin{array}{l}\mathrm{E}=35.2 \pm 7.4 \\
\mathrm{C}=34.9 \pm 8.5\end{array}$ & $30 / 30$ & $\begin{array}{l}\text { Staff hospital }{ }^{1} \text { Administrative } \\
\text { employees }\end{array}$ & n.a. & $\begin{array}{l}\text { Gloves, caps, overalls, } \\
\text { goggles }\end{array}$ & lymphocytes & $\begin{array}{c}10.72 \pm 7.04 \\
12.32 \pm 10.06\end{array}$ & 18 \\
\hline Cavallo D. et al., 2009 (b) & Italy & $\mathrm{M}+\mathrm{F}$ & $\begin{array}{l}\mathrm{E}=35.2 \pm 7.4 \\
\mathrm{C}=34.9 \pm 8.5\end{array}$ & $30 / 30$ & $\begin{array}{l}\text { Staff hospital }{ }^{1} \text { Administrative } \\
\text { employees }\end{array}$ & n.a. & $\begin{array}{l}\text { Gloves, caps, overalls, } \\
\text { goggles }\end{array}$ & buccal & $\begin{array}{l}12.00 \pm 6.10 \\
14.45 \pm 11.7\end{array}$ & 18 \\
\hline Connor T. et al., 2010 & USA & $\mathrm{M}+\mathrm{F}$ & $\begin{array}{l}\mathrm{E}=38.5 \pm 10.5 \\
\mathrm{C}=39.9 \pm 10.4\end{array}$ & $68 / 53$ & $\begin{array}{l}\text { Staff hospital }{ }^{2} \text { involved in } \\
\text { oncological wards } \\
\text { Staff hospital not involved in } \\
\text { oncological wards }\end{array}$ & n.a. & $\begin{array}{l}\text { Gloves, gowns, goggles, } \\
\text { masks, vertical air-flow } \\
\text { cabinet }\end{array}$ & lymphocytes & $\begin{array}{l}53.06 \pm 7.32 \\
53.12 \pm 7.5\end{array}$ & 17 \\
\hline Cornetta T. et al., 2008 (a) & Italy & $\mathrm{M}+\mathrm{F}$ & $\begin{array}{l}E=37.6 \pm 6.7 \\
C=37.0 \pm 10.0\end{array}$ & $83 / 73$ & $\begin{array}{c}\text { Hospital nurses } \\
\text { Administrative employees }\end{array}$ & $12.2 \pm 7.3$ & $\begin{array}{l}\text { Gloves, overalls, } \\
\text { goggles masks, vertical } \\
\text { air-flow cabinet }\end{array}$ & lymphocytes & $\begin{array}{l}1.16 \pm 0.82 \\
0.77 \pm 0.47\end{array}$ & 17 \\
\hline Cornetta T. et al., 2008 (b) & Italy & M & n.a. & $16 / 20$ & $\begin{array}{c}\text { Hospital nurses } \\
\text { Administrative employees }\end{array}$ & $12.2 \pm 7.3$ & $\begin{array}{l}\text { Gloves, overalls, } \\
\text { goggles masks, vertical } \\
\text { air-flow cabinet }\end{array}$ & lymphocytes & $\begin{array}{l}1.13 \pm 0.98 \\
0.88 \pm 0.45\end{array}$ & 17 \\
\hline Cornetta T. et al., 2008 (c) & Italy & $\mathrm{F}$ & n.a. & $67 / 53$ & $\begin{array}{c}\text { Hospital nurses } \\
\text { Administrative employees }\end{array}$ & $12.2 \pm 7.3$ & $\begin{array}{l}\text { Gloves, overalls, } \\
\text { goggles masks, vertical } \\
\text { air-flow cabinet }\end{array}$ & lymphocytes & $\begin{array}{l}1.16 \pm 0.78 \\
0.73 \pm 0.48\end{array}$ & 17 \\
\hline Hongping D. et al., 2005 (a) & China & $\mathrm{M}+\mathrm{F}$ & $\begin{array}{l}\mathrm{E}=35.0 \pm 10.4 \\
\mathrm{C}=36.4 \pm 10.0\end{array}$ & $21 / 21$ & $\begin{array}{l}\text { Drug technicians } \\
\text { n.a. }\end{array}$ & $5.6 \pm 4.2$ & Gloves, masks & lymphocytes & $\begin{array}{l}1.30 \pm 0.29^{\circ} \\
0.70 \pm 0.03^{\circ}\end{array}$ & 16 \\
\hline Hongping D. et al., 2005 (b) & China & M & $\begin{array}{l}\mathrm{E}=32.0 \pm 10.9 \\
\mathrm{C}=33.0 \pm 10.4\end{array}$ & $11 / 11$ & $\begin{array}{l}\text { Drug technicians } \\
\text { n.a. }\end{array}$ & $4.3 \pm 3.2$ & Gloves, masks & lymphocytes & $\begin{array}{l}1.35 \pm 0.18^{\circ} \\
0.69 \pm 0.02 \S\end{array}$ & 16 \\
\hline Hongping D. et al., 2005 (c) & China & $\mathrm{F}$ & $\begin{array}{l}\mathrm{E}=38.4 \pm 9.2 \\
\mathrm{C}=40.1 \pm 8.6\end{array}$ & $10 / 10$ & $\begin{array}{l}\text { Drug technicians } \\
\text { n.a. }\end{array}$ & $7.0 \pm 4.8$ & Gloves, masks & lymphocytes & $\begin{array}{l}1.26 \pm 0.38^{\circ} \\
0.72 \pm 0.04 \S\end{array}$ & 16 \\
\hline Hongping D. et al., 2006 (a) & China & $\mathrm{M}+\mathrm{F}$ & $\begin{array}{l}\mathrm{E}=43.7 \pm 1.1^{\wedge} \\
\mathrm{C}=43.5 \pm 1.4^{\wedge}\end{array}$ & $15 / 15$ & $\begin{array}{l}\text { Drug technicians } \\
\text { n.a. }\end{array}$ & $7.1 \pm 4.35$ & Gloves, masks & lymphocytes & $\begin{array}{l}1.72 \pm 0.57^{\circ} \\
0.71 \pm 0.04^{\circ}\end{array}$ & 14 \\
\hline Hongping D. et al., 2006 (b) & China & M & $\begin{array}{l}\mathrm{E}=44.2 \pm 2.4^{\wedge} \\
\mathrm{C}=42.2 \pm 2.9^{\wedge}\end{array}$ & 6/6 & $\begin{array}{l}\text { Drug technicians } \\
\text { n.a. }\end{array}$ & $5.8 \pm 2.8$ & Gloves, masks & lymphocytes & $\begin{array}{l}1.88 \pm 0.66 \S \\
0.71 \pm 0.03 \S\end{array}$ & 14 \\
\hline Hongping D. et al., 2006 (c) & China & $\mathrm{F}$ & $\begin{array}{l}\mathrm{E}=43.3 \pm 1.1^{\wedge} \\
\mathrm{C}=44.3 \pm 1.4^{\wedge}\end{array}$ & 9/9 & $\begin{array}{l}\text { Drug technicians } \\
\text { n.a. }\end{array}$ & $7.9 \pm 5.1$ & Gloves, masks & lymphocytes & $\begin{array}{l}1.62 \pm 0.52 \S \\
0.71 \pm 0.05 \S\end{array}$ & 14 \\
\hline Izdes A. et al., 2009 & Turkey & $\mathrm{M}+\mathrm{F}$ & $\begin{array}{l}\mathrm{E}=32.3 \pm 5.9 \\
\mathrm{C}=33.5 \pm 5.1\end{array}$ & 19/19 & $\begin{array}{c}\text { Hospital nurses } \\
\text { Administrative employees }\end{array}$ & $11.3 \pm 4.2$ & $\begin{array}{l}\text { Gloves, masks, vertical } \\
\text { air-flow cabinet }\end{array}$ & lymphocytes & $\begin{array}{c}19.89 \pm 4.84 \\
6.84 \pm 3.16\end{array}$ & 14 \\
\hline Kopjar N. Garaj-Vrhovac V. 2001 (a) & Croatia & $\mathrm{F}$ & $\begin{array}{l}\mathrm{E}=37.0 \pm 8.9 \\
\mathrm{C}=29.5 \pm 8.2\end{array}$ & $50 / 20$ & $\begin{array}{c}\text { Hospital nurses } \\
\text { Students, administrative } \\
\text { employees }\end{array}$ & $12.9 \pm 9.4$ & $\begin{array}{l}\text { Gloves, masks, vertical } \\
\text { air-flow cabinet }\end{array}$ & lymphocytes & $\begin{array}{l}81.49 \pm 4.31 \\
76.01 \pm 3.7\end{array}$ & 12 \\
\hline Kopjar N. Garaj-Vrhovac V. 2001 (b) & Croatia & $\mathrm{F}$ & $\begin{array}{l}\mathrm{E}=36.5 \pm 9.5 \\
\mathrm{C}=29.5 \pm 8.2\end{array}$ & 20/20 & $\begin{array}{l}\text { Hospital nurses } \\
\text { Students, administrative } \\
\text { employees }\end{array}$ & $12.1 \pm 9.5$ & Gloves & lymphocytes & $\begin{array}{c}83.44 \pm 1.49 \\
76.01 \pm 3.7\end{array}$ & 12 \\
\hline Kopjar N. Garaj-Vrhovac V. 2001 (c) & Croatia & $\mathrm{F}$ & $\begin{array}{l}\mathrm{E}=35.5 \pm 9.3 \\
\mathrm{C}=29.5 \pm 8.2\end{array}$ & $8 / 20$ & $\begin{array}{l}\text { Hospital nurses } \\
\text { Students, administrative } \\
\text { employees }\end{array}$ & $14.1 \pm 8.8$ & Gloves, masks & lymphocytes & $\begin{array}{l}81.6 \pm 4.51 \\
76.01 \pm 3.7\end{array}$ & 12 \\
\hline Kopjar N. Garaj-Vrhovac V. 2001 (d) & Croatia & $\mathrm{F}$ & $\begin{array}{l}\mathrm{E}=37.8 \pm 8.4 \\
\mathrm{C}=29.5 \pm 8.2\end{array}$ & 19/20 & $\begin{array}{l}\text { Hospital nurses } \\
\text { Students, administrative } \\
\text { employees }\end{array}$ & $13.0 \pm 9.9$ & $\begin{array}{l}\text { Gloves, vertical air-flow } \\
\text { cabinet }\end{array}$ & lymphocytes & $\begin{array}{l}80.14 \pm 5.17 \\
76.01 \pm 3.7\end{array}$ & 12 \\
\hline Kopjar N. Garaj-Vrhovac V. 2001 (e) & Croatia & $\mathrm{F}$ & $\begin{array}{l}E=39.3 \pm 10.1 \\
C=29.5 \pm 8.2\end{array}$ & $3 / 20$ & $\begin{array}{l}\text { Hospital nurses } \\
\text { Students, administrative } \\
\text { employees }\end{array}$ & $14 \pm 12.5$ & $\begin{array}{l}\text { Gloves, masks, vertical } \\
\text { air-flow cabinet }\end{array}$ & lymphocytes & $\begin{array}{c}76.8 \pm 5.9 \\
76.01 \pm 3.7\end{array}$ & 12 \\
\hline
\end{tabular}


Table 2. Cont.

\begin{tabular}{|c|c|c|c|c|c|c|c|c|c|c|}
\hline Author, Year & Country & Gender & $\begin{array}{c}\text { Mean Age } \pm \text { SD } \\
\text { (Years) }\end{array}$ & $n(\mathrm{E} / \mathrm{C}) \ddagger$ & Work Task & $\begin{array}{l}\text { Mean Exposure } \pm \\
\text { SD (Years) }\end{array}$ & Protective Equipment & Cells & $\begin{array}{l}\text { Comet Test } \\
\text { Mean } \pm \text { SD }\end{array}$ & $\mathrm{QS} / 27$ \\
\hline Kopjar N. et al., 2009 & Croatia & $\mathrm{F}$ & $\begin{array}{l}\mathrm{E}=37.0 \pm 8.9 \\
\mathrm{C}=37.9 \pm 8.9\end{array}$ & $50 / 50$ & $\begin{array}{c}\text { Staff hospital }{ }^{3} \\
\text { Students, administrative } \\
\text { employees }\end{array}$ & $12.9 \pm 9.4$ & $\begin{array}{l}\text { Gloves, masks, vertical } \\
\text { air-flow cabinet }\end{array}$ & lymphocytes & $\begin{array}{r}17.46 \pm 1.99 \\
14.00 \pm 0.14^{\circ}\end{array}$ & 17 \\
\hline Ladeira C. et al., 2015 & Portugal & $\mathrm{M}+\mathrm{F}$ & $\begin{array}{l}\mathrm{E}=33.8 \pm 1.2^{\wedge} \\
\mathrm{C}=39.3 \pm 1.4\end{array}$ & $46 / 46$ & $\begin{array}{l}\text { Staff hospital }{ }^{4} \\
\text { Teachers, administrative } \\
\text { employees }\end{array}$ & $6.6 \pm 0.9 *$ & n.a. & lymphocytes & $\begin{array}{l}13.36^{*} \\
11.12 *\end{array}$ & 15 \\
\hline Laffon B. et al., 2005 (a) & Portugal & $\mathrm{M}+\mathrm{F}$ & $\begin{array}{l}\mathrm{E}=33,3 \pm 9,2 \\
\mathrm{C}=44,1 \pm 8,2\end{array}$ & 29/22 & $\begin{array}{l}\text { Hospital nurses } \\
\text { Hospital nurses }\end{array}$ & $6.4 \pm 6.2$ & $\begin{array}{c}\text { Wearing laboratory } \\
\text { coat, mask, gloves } \\
\text { vertical air-flow cabinet }\end{array}$ & lymphocytes & $\begin{array}{c}46.46 \pm 0.48 \\
\quad \text { o } \\
42.68 \pm 0.47 \\
\text { 。 }\end{array}$ & 14 \\
\hline Laffon B. et al., 2005 (b) & Portugal & M & n.a. & $4 / 2$ & $\begin{array}{l}\text { Hospital nurses } \\
\text { Hospital nurses }\end{array}$ & $6.4 \pm 6.2$ & $\begin{array}{c}\text { Wearing laboratory } \\
\text { coat, mask, gloves } \\
\text { vertical air-flow cabinet }\end{array}$ & lymphocytes & $\begin{array}{c}46.65 \pm 0.40 \\
\stackrel{4}{\circ} \\
42.74 \pm 0.41\end{array}$ & 14 \\
\hline Laffon B. et al., 2005 (c) & Portugal & $\mathrm{F}$ & n.a. & $25 / 20$ & $\begin{array}{l}\text { Hospital nurses } \\
\text { Hospital nurses }\end{array}$ & $6.4 \pm 6.2$ & $\begin{array}{c}\text { Wearing laboratory } \\
\text { coat, mask, gloves } \\
\text { vertical air-flow cabinet }\end{array}$ & lymphocytes & $\begin{array}{c}46.43 \pm 0.50 \\
\stackrel{0}{\circ} \\
42.67 \pm 0.49 \\
0\end{array}$ & 14 \\
\hline Maluf S.W. Anda Erdtmann B. 2000 & Brazil & $\mathrm{M}+\mathrm{F}$ & $\begin{array}{l}\mathrm{E}=34.7 \pm 5.4 \\
\mathrm{C}=34.4 \pm 4.5\end{array}$ & $12 / 12$ & $\begin{array}{c}\text { Staff hospital } \\
n \text { n.a. }\end{array}$ & $3.4 \pm 2.0$ & n.a. & lymphocytes & $\begin{array}{c}20.83 \pm 10.19 \\
8.08 \pm 5.16\end{array}$ & 15 \\
\hline Rekhadevi P.V. et al., 2007 & India & $\mathrm{F}$ & $\begin{array}{l}\mathrm{E}=38.2 \pm 5.6 \\
\mathrm{C}=37.9 \pm 5.6\end{array}$ & 60/60 & $\begin{array}{l}\text { Hospital nurses } \\
\text { General population }\end{array}$ & $13.6 \pm 4.8$ & n.a. & lymphocytes & $\begin{array}{r}13.66 \pm 2.37 \\
6.21 \pm 0.92\end{array}$ & 19 \\
\hline Rombaldi F. et al., 2009 & Brazil & $\mathrm{M}+\mathrm{F}$ & $\begin{array}{l}E=31.5 \pm 9.3 \\
C=28.2 \pm 6.3\end{array}$ & $20 / 20$ & $\begin{array}{l}\text { Staff hospital } \\
\text { General population }\end{array}$ & $2.9 \pm 3.0$ & $\begin{array}{c}\text { Wearing laboratory } \\
\text { coat, mask, gloves } \\
\text { vertical air-flow cabinet }\end{array}$ & lymphocytes & $\begin{array}{c}18.86 \pm 8.62 \\
6.21 \pm 2.78\end{array}$ & 18 \\
\hline Sasaki M. et al., 2008 & Japan & F & $\begin{array}{l}\mathrm{E}=37.0 \pm 10.0 \\
\mathrm{C}=36.0 \pm 9.0\end{array}$ & $121 / 46$ & $\begin{array}{l}\text { Hospital nurses } \\
\text { Clerks hospital }\end{array}$ & n.a. & n.a. & lymphocytes & $\begin{array}{l}0.764 \pm 0.121 \\
0.711 \pm 0.089\end{array}$ & 16 \\
\hline Undeger U. et al., 1999 & Turkey & $\mathrm{M}+\mathrm{F}$ & $\begin{array}{l}\mathrm{E}=29.0 \pm 5.0 \\
\mathrm{C}=29.0 \pm 5.0\end{array}$ & $30 / 30$ & $\begin{array}{l}\text { Hospital nurses } \\
\text { Staff hospital (secretaries, } \\
\text { nurses techinicians) }\end{array}$ & $3.8 \pm 3.1$ & $\begin{array}{l}\text { Gloves, masks, gowns, } \\
\text { eye glasses caps }\end{array}$ & lymphocytes & $\begin{array}{l}105.05 \pm 36.0 \\
153.8 \pm 18.3\end{array}$ & 19 \\
\hline Ursini C.L. et al., 2006 (a) & Italy & $\mathrm{M}+\mathrm{F}$ & $\begin{array}{l}E=35.8 \pm 9.9 \\
C=34.9 \pm 8.5\end{array}$ & $5 / 30$ & $\begin{array}{l}\text { Staff hospital }{ }^{5} \\
\text { Hospital employees }\end{array}$ & $7.0 \pm 2.0$ & $\begin{array}{l}\text { Gloves, caps, overalls, } \\
\text { goggles }\end{array}$ & lymphocytes & $\begin{array}{l}20.8 \pm 10.1 \\
16.1 \pm 8.1\end{array}$ & 18 \\
\hline Ursini C.L. et al., 2006 (b) & Italy & $\mathrm{M}+\mathrm{F}$ & $\begin{array}{l}\mathrm{E}=37.6 \pm 5.5 \\
\mathrm{C}=34.9 \pm 8.5\end{array}$ & $12 / 30$ & $\begin{array}{l}\text { Staff hospital }{ }^{5} \\
\text { Hospital employees }\end{array}$ & $8.1 \pm 6.0$ & $\begin{array}{l}\text { Gloves, caps, overalls, } \\
\text { goggles }\end{array}$ & lymphocytes & $\begin{array}{l}15.5 \pm 9.0 \\
16.1 \pm 8.1\end{array}$ & 18 \\
\hline Ursini C.L. et al., 2006 (c) & Italy & $\mathrm{M}+\mathrm{F}$ & $\begin{array}{l}\mathrm{E}=32.7 \pm 7.7 \\
\mathrm{C}=34.9 \pm 8.5\end{array}$ & $13 / 30$ & $\begin{array}{l}\text { Staff hospital }^{5} \\
\text { Hospital employees }\end{array}$ & $6.2 \pm 2.9$ & $\begin{array}{l}\text { Gloves, caps, overalls, } \\
\text { goggles }\end{array}$ & lymphocytes & $\begin{array}{l}14.7 \pm 7.9 \\
16.1 \pm 8.1\end{array}$ & 18 \\
\hline Ursini C.L. et al., 2006 (d) & Italy & $\mathrm{M}+\mathrm{F}$ & $\begin{array}{l}\mathrm{E}=35.8 \pm 9.9 \\
\mathrm{C}=34.9 \pm 8.5\end{array}$ & $5 / 30$ & $\begin{array}{l}\text { Staff hospital }^{5} \\
\text { Hospital employees }\end{array}$ & $7.0 \pm 2.0$ & $\begin{array}{l}\text { Gloves, caps, overalls, } \\
\text { goggles }\end{array}$ & buccal & $\begin{array}{l}32.6 \pm 18.2 \\
28.6 \pm 12.4\end{array}$ & 18 \\
\hline Ursini C.L. et al., 2006 (d) & Italy & $\mathrm{M}+\mathrm{F}$ & $\begin{array}{l}E=37.6 \pm 5.5 \\
C=34.9 \pm 8.5\end{array}$ & $12 / 30$ & $\begin{array}{l}\text { Staff hospital }{ }^{5} \\
\text { Hospital employees }\end{array}$ & $8.1 \pm 6.0$ & $\begin{array}{l}\text { Gloves, caps, overalls, } \\
\text { goggles }\end{array}$ & buccal & $\begin{array}{l}43.2 \pm 36.0 \\
28.6 \pm 12.4\end{array}$ & 18 \\
\hline Ursini C.L. et al., 2006 (e) & Italy & $\mathrm{M}+\mathrm{F}$ & $\begin{array}{l}\mathrm{E}=32.7 \pm 7.7 \\
\mathrm{C}=34.9 \pm 8.5\end{array}$ & $13 / 30$ & $\begin{array}{l}\text { Staff hospital }{ }^{5} \\
\text { Hospital employees }\end{array}$ & $6.2 \pm 2.9$ & $\begin{array}{l}\text { Gloves, caps, overalls, } \\
\text { goggles }\end{array}$ & buccal & $\begin{array}{l}27.4 \pm 13.9 \\
28.6 \pm 12.4\end{array}$ & 18 \\
\hline Villarini M. et al., 2011 (a) & Italy & $\mathrm{M}+\mathrm{F}$ & $\begin{array}{l}\mathrm{E}=39.3 \pm 9.6 \\
\mathrm{C}=36.2 \pm 11.2\end{array}$ & $52 / 52$ & $\begin{array}{l}\text { Staff hospital } \\
\text { Hospital employees }\end{array}$ & n.a. & Gloves, masks & lymphocytes & $\begin{array}{l}2.73 \pm 2.02^{\circ} \\
1.67 \pm 1.01^{\circ}\end{array}$ & 18 \\
\hline Villarini M. et al., 2011 (b) & Italy & M & n.a. & $7 / 12$ & $\begin{array}{c}\text { Staff hospital }^{5} \\
\text { Hospital employees }\end{array}$ & n.a. & Gloves, masks & lymphocytes & $\begin{array}{l}1.82 \pm 0.74^{\circ} \\
1.76 \pm 1.42^{\circ}\end{array}$ & 18 \\
\hline Villarini M. et al., 2011 (c) & Italy & $\mathrm{F}$ & n.a. & $45 / 40$ & $\begin{array}{l}\text { Staff hospital }^{5} \\
\text { Hospital employees }\end{array}$ & n.a. & Gloves, masks & lymphocytes & $\begin{array}{l}2.86 \pm 2.08^{\circ} \\
1.64 \pm 0.95^{\circ}\end{array}$ & 18 \\
\hline Yoshida J. et al., 2006 & Japan & $\mathrm{F}$ & $\begin{array}{l}E=29.2 \\
C=31.6\end{array}$ & 19/18 & $\begin{array}{l}\text { Hospital nurses } \\
\text { Hospital nurses }\end{array}$ & 5.7 & Gloves, masks & lymphocytes & $\begin{array}{l}8.80 \pm 2.27 \\
6.60 \pm 1.07\end{array}$ & 16 \\
\hline
\end{tabular}

$\ddagger \mathrm{E}=$ exposed, $\mathrm{C}=$ controls, ${ }^{\wedge} \pm \mathrm{SE} ;{ }^{*}$ median; ${ }^{\circ} \mathrm{SD}$ estimated from SEM; ${ }^{\S}$ mean and SD estimated from SEM $;{ }^{1}$ staff hospital (nurses, drug technicians); ${ }^{2}$ staff hospital (nurses, pharmacists drug technicians, nursing assistants); ${ }^{3}$ staff hospital (nurses, medical); ${ }^{4}$ staff hospital (nurses, pharmacists, drug technicians); ${ }^{5}$ staff hospital (nurses, pharmacists); $\mathrm{M}$, male; $\mathrm{F}$, female; $\mathrm{QS}$, quality score. 


\subsection{Characteristics of the Studied Populations}

The sample size of the included studies ranged between 12 and 83 exposed subjects, and 12 and 74 controls. The mean age and SD of the exposed subjects were between $29 \pm 5$ and $44.17 \pm 2.40$ years, while for controls, they were $28.23 \pm 6.30$ and $44.33 \pm 1.41$ years. The exposed and control groups were perfectly matched for age $(n=3)$, gender $(n=9)$, and smoking $(n=6)$ in several studies. Considering the nutritional intake, a very large majority of the studies either did not match the two groups $(n=18)$, or only partially matched $(n=2)$ by taking into account this confounding variable. In all studies, the considered exposed subjects were nurses, whereas in two studies [34,35], they were the workers producing ANDs. Moreover, pharmacists or pharmacy technicians were also included in the exposed group. Only one study considered physicians as well [38]. Most of the time, the controls comprised workers not occupationally exposed to ANDs. In five studies, the controls were nurses not involved in oncological wards [30,32,40,44,48], while in two studies, no details regarding the characteristics of control subjects were provided [34,35]. Most of the time, exposure to ANDs was assessed through a self-reported questionnaire; environmental monitoring was included in the study design in four studies [39,47-49], whereas only three studies [32,42,46] performed biological measurements. Regarding personal protective equipment, gloves and masks were the most frequently used; wearing lab coats, glasses, and caps was also reported. Among the environmental equipment, the use of an air-flow cabinet was reported in only seven studies (Table 2).

Approximately half of the included studies $(n=9)$ scored 100 cells per subject, and eight studies scored a number of cells between 100 and 200; lastly, three studies scored more than 200 cells per subject. The shape of a comet tail represents the entity of DNA damage; the higher the migration of chromosomal DNA from the nucleus, the higher the DNA damage. Several units of measure are used to express the results. In the retrieved studies, tail intensity $\%$ was the most used $(n=7)$, followed by tail length $(n=6)$. The DNA damage index was used in three studies; the tail moment, the total comet score, and the log tail length were used once, respectively. Lastly, one study considered a tail factor without any other further explanation [49]. However, all the results were expressed as a mean and SD or standard error of mean (SEM). One study expressed the results as the median [39], and one reported both mean and median [48].

\subsection{Results of the Meta-Analysis}

Considering the comet assay performed on peripheral blood lymphocytes, a total of 19 studies were included in the quantitative evaluation. However, one study reported the data separately for pharmacists, nurses working in day hospital, and nurses working in the ward, so they were considered as three independent studies and, for this reason, the meta-analysis included 21 datasets. The pooled ES was 1.27 [ $(95 \% \mathrm{CI}=0.66-1.88), p$-value $<0.001]$ based on 1569 subjects (Figure 2a), with high statistical heterogeneity $\left(\mathrm{Chi}^{2}=541.17, \mathrm{df}=20, \mathrm{I}^{2}=96.30, p\right.$-value $\left.<0.001\right)$. A potential publication bias was found by the visual assessment of the funnel plot and confirmed by Egger's linear regression test (intercept 7.56, $\mathrm{t}=2.88, p$-value $=0.010$. However, the estimated ES did not change after the trim and fill method was applied (Figure $2 b$ ). The publication year plot is depicted in Figure 2c, showing the sequence of publication. 


\begin{tabular}{rccccr} 
& ES & 95\% Cl & W & Sig. & \multicolumn{1}{c}{$\mathbf{N}$} \\
Laffon (a) 2005 & 7.95 & $6.31,9.58$ & $3.74 \%$ & 0.000 & 51 \\
Rekhadevi 2007 & 4.14 & $3.51,4.78$ & $4.83 \%$ & 0.000 & 120 \\
Izdes 2009 & 3.19 & $2.23,4.15$ & $4.53 \%$ & 0.000 & 38 \\
Hongping (a) 2005 & 2.91 & $2.04,3.78$ & $4.62 \%$ & 0.000 & 42 \\
Hongping (a) 2006 & 2.50 & $1.54,3.45$ & $4.53 \%$ & 0.000 & 30 \\
kopjar 2009 & 2.45 & $1.93,2.97$ & $4.91 \%$ & 0.000 & 100 \\
Rombaldi 2009 & 1.98 & $1.22,2.73$ & $4.73 \%$ & 0.000 & 40 \\
Maluf 2000 & 1.58 & $0.66,2.49$ & $4.57 \%$ & 0.001 & 24 \\
Yoshida 2006 & 1.56 & $0.83,2.30$ & $4.74 \%$ & 0.000 & 37 \\
Kopjar (a) 2001 & 1.32 & $0.76,1.88$ & $4.88 \%$ & 0.000 & 70 \\
Villarini (a) 2011 & 0.66 & $0.27,1.06$ & $4.99 \%$ & 0.001 & 104 \\
Cornetta (a) 2008 & 0.57 & $0.25,0.90$ & $5.02 \%$ & 0.000 & 156 \\
Ursini (a) 2006 & 0.56 & $-0.39,1.52$ & $4.53 \%$ & 0.249 & 35 \\
Sasaki 2008 & 0.47 & $0.13,0.81$ & $5.01 \%$ & 0.007 & 167 \\
Ladeira 2015 & 0.31 & $-0.10,0.72$ & $4.98 \%$ & 0.135 & 92 \\
Connor 2010 & -0.01 & $-0.37,0.35$ & $5.00 \%$ & 0.965 & 121 \\
Ursini (b) 2006 & -0.07 & $-0.74,0.60$ & $4.80 \%$ & 0.834 & 42 \\
Ursini (c) 2006 & -0.17 & $-0.83,0.48$ & $4.81 \%$ & 0.601 & 43 \\
Cavallo (a) 2009 & -0.18 & $-0.69,0.32$ & $4.92 \%$ & 0.476 & 60 \\
Buschini 2013 & -1.33 & $-1.70,-0.96$ & $5.00 \%$ & 0.000 & 137 \\
Undeger 1999 1996 & -1.71 & $-2.30,-1.12$ & $4.86 \%$ & 0.000 & 60 \\
Overall (random-effects model) & 1.27 & $0.66,1.88$ & $100.00 \%$ & 0.000 & 1569
\end{tabular}

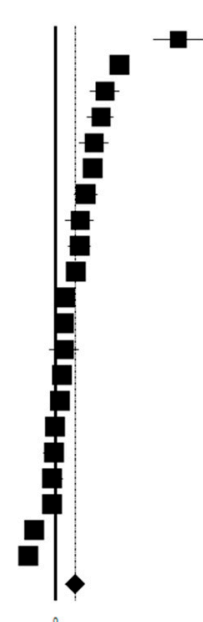

(a)

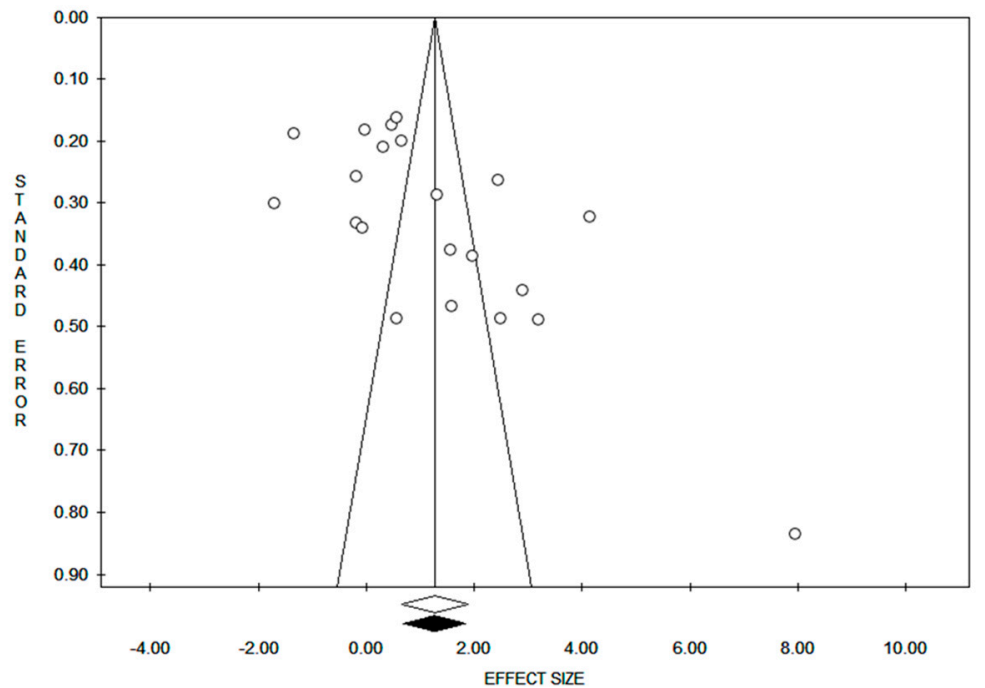

(b)

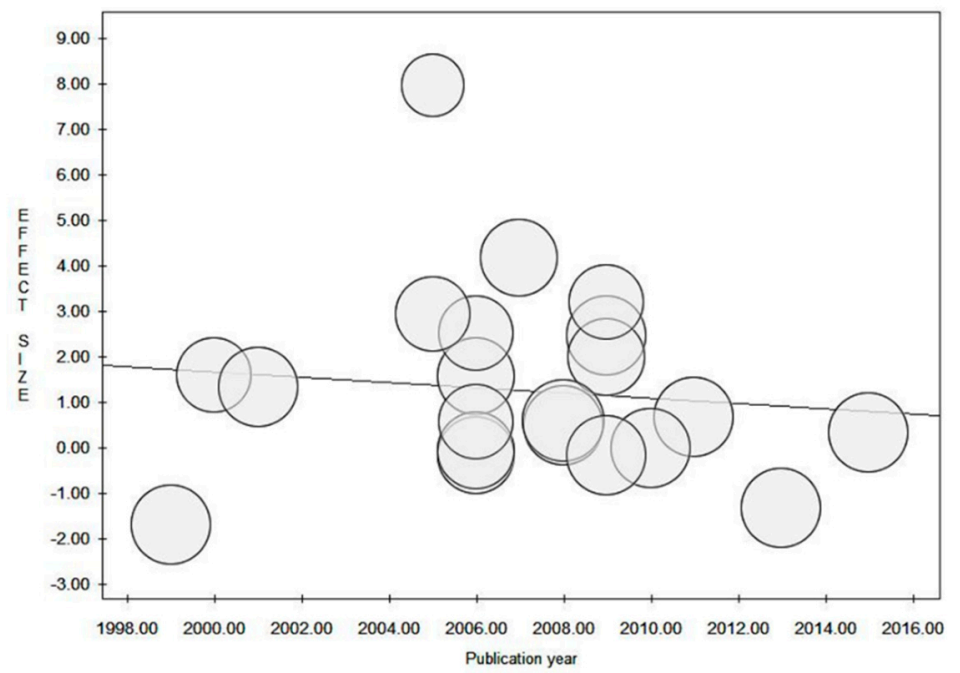

(c)

Figure 2. (a) Forest plot, (b) funnel plot, and (c) publication year plot of the meta-analysis assessing the comet assay on lymphocytes cells among professionals occupationally exposed to antineoplastic drugs (ANDs). ES, effect size; CI, confidence interval. 
Considering the comet assay performed on buccal cells, a total of four datasets were included. The pooled ES was 0.10 [(95\% CI $=-0.34-0.54), p$-value $=0.652]$ based on 180 subjects (Figure $3 a$ ). Low statistical heterogeneity was found $\left(\mathrm{Chi}^{2}=5.11, \mathrm{df}=4, \mathrm{I}^{2}=41.27, p\right.$-value $\left.=0.164\right)$. No publication bias was found by the visual assessment of the funnel plot, and confirmed by Egger's linear regression test (intercept 3.33, $\mathrm{t}=1.12, p$-value $=0.378$ ) (Figure $3 \mathrm{~b}$ ).

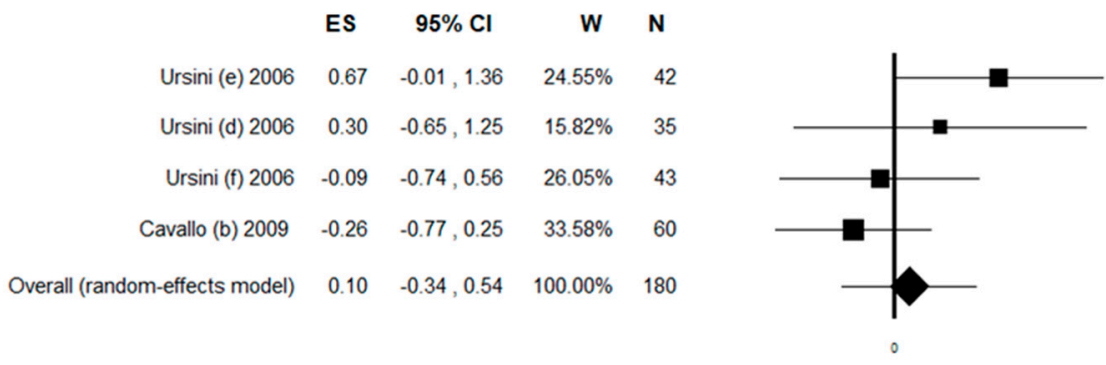

(a)

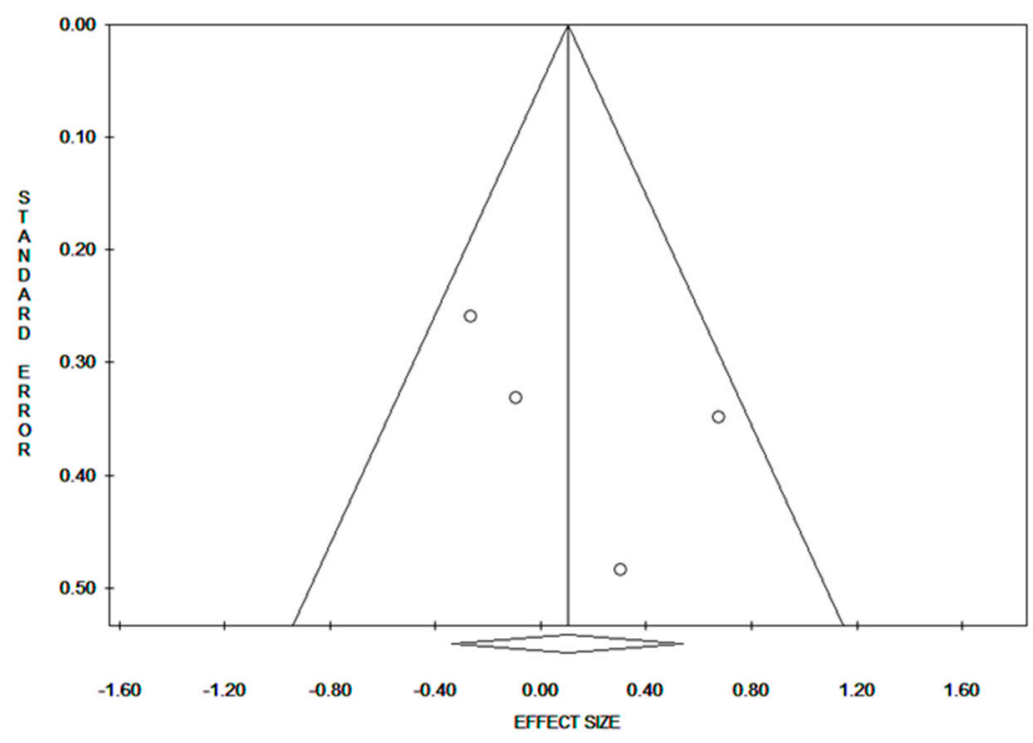

(b)

Figure 3. (a) Forest plot and (b) funnel plot of the meta-analysis assessing the comet assay on buccal cells among professionals occupationally exposed to antineoplastic drugs (ANDs).

\subsection{Sensitivity Analysis by Quality Score}

In order to reduce heterogeneity, only studies with $\mathrm{QS} \geq 16$ were included. The pooled ES was $0.92[(95 \% \mathrm{CI}=0.26-1.59), p$-value $=0.006]$ based on 1127 subjects (Figure $4 \mathrm{a})$. A high statistical heterogeneity was found $\left(\mathrm{Chi}^{2}=302.73, \mathrm{df}=13, \mathrm{I}^{2}=95.71, p\right.$-value $\left.<0.001\right)$. No potential publication bias was found by visual assessment of the funnel plot, and confirmed by Egger's linear regression test (intercept $4.22, \mathrm{t}=1.08, p$-value $=0.300)($ Figure $4 \mathrm{~b})$. 


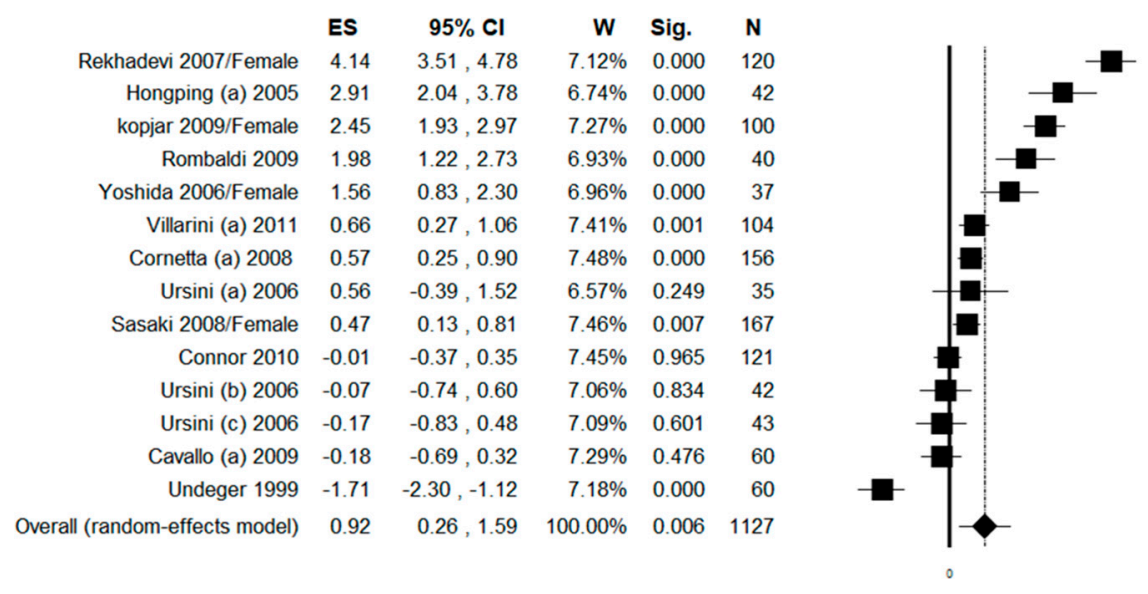

(a)

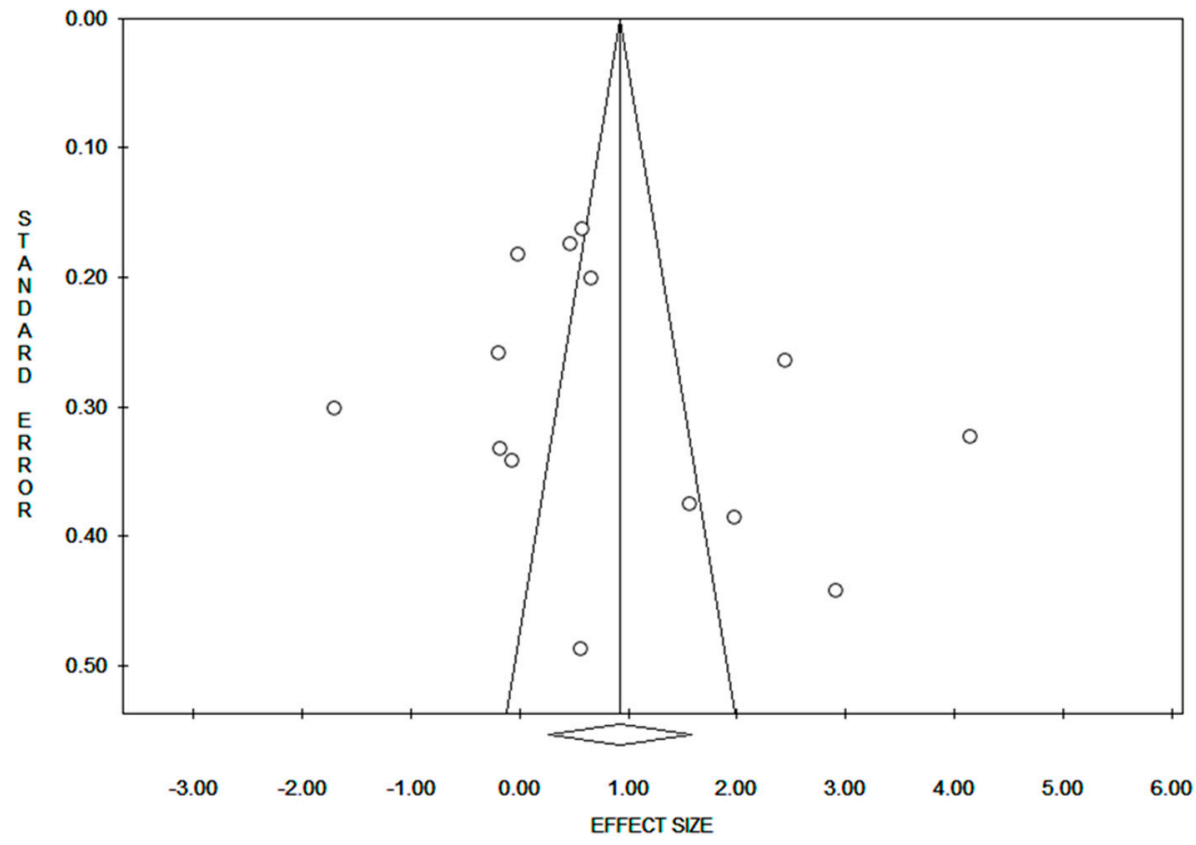

(b)

Figure 4. (a) Forest plot and (b) funnel plot (after trim and fill method) of the meta-analysis assessing the comet assay on lymphocytes cells among professionals occupationally exposed to antineoplastic drugs (ANDs), only including studies with a quality score (QS) $\geq 16$.

\subsection{Sub-Group Analysis by Gender}

In order to increase the robustness of the results, a sub-group analysis by gender was performed. The sub-group analysis considering only females included 10 datasets, and the pooled ES was 1.95 [(95\% CI $=0.88-3.02), p$-value $<0.001]$ based on 849 subjects (Figure 5). High statistical heterogeneity was found $\left(\mathrm{Chi}^{2}=355.84, \mathrm{df}=9, \mathrm{I}^{2}=97.47, p\right.$-value $\left.<0.001\right)$. The sub-group analysis considering only males included five studies and the pooled ES was 2.50 [ ( $95 \% \mathrm{CI}=0.57-4.44), p$-value $=0.011]$ based on 95 subjects (Figure 5). High statistical heterogeneity was found $\left(\mathrm{Chi}^{2}=42.31, \mathrm{df}=4, \mathrm{I}^{2}=90.55\right.$, $p$-value $<0.001$ ). Potential publication bias was found by visual assessment of the funnel plot, and confirmed by Egger's linear regression test (intercept 7.28, $\mathrm{t}=3.15, p$-value $=0.008$ ). However, after applying the trim and fill method, the estimated ES did not change. 


$\begin{array}{rcrrrr} & \text { ES } & \text { 95\% Cl } & \text { W } & \text { Sig. } & \text { N } \\ \text { Laffon (c) 2005 } & 7.59 & 5.91,9.26 & 8.43 \% & 0.000 & 45 \\ \text { Rekhadevi 2007 } & 4.14 & 3.51,4.78 & 10.24 \% & 0.000 & 120 \\ \text { Hongping (c) 2006 } & 2.46 & 1.24,3.69 & 9.33 \% & 0.000 & 18 \\ \text { kopjar 2009 } & 2.45 & 1.93,2.97 & 10.36 \% & 0.000 & 100 \\ \text { Hongping (c) 2005 } & 2.00 & 0.93,3.07 & 9.60 \% & 0.000 & 20 \\ \text { Yoshida 2006 } & 1.56 & 0.83,2.30 & 10.11 \% & 0.000 & 37 \\ \text { Villarini (c) 2011 } & 0.74 & 0.30,1.18 & 10.44 \% & 0.001 & 85 \\ \text { Cornetta (c) 2008 } & 0.65 & 0.28,1.02 & 10.49 \% & 0.001 & 120 \\ \text { Sasaki 2008 } & 0.47 & 0.13,0.81 & 10.51 \% & 0.007 & 167 \\ \text { Buschini 2013 } & -1.33 & -1.70,-0.96 & 10.49 \% & 0.000 & 137 \\ \text { F } & 1.95 & 0.88,3.02 & 100.00 \% & 0.000 & 849 \\ & & & & & \\ \text { Laffon (b) 2005 } & 9.71 & 3.96,15.47 & 7.83 \% & 0.001 & 6 \\ \text { Hongping (b) 2005 } & 5.15 & 3.42,6.89 & 21.17 \% & 0.000 & 22 \\ \text { Hongping (b) 2006 } & 2.50 & 0.99,4.02 & 22.09 \% & 0.001 & 12 \\ \text { Cornetta (b) 2008 } & 0.34 & -0.32,1.00 & 24.80 \% & 0.312 & 36 \\ \text { Villarini (b) 2011 } & 0.05 & -0.88,0.98 & 24.11 \% & 0.918 & 19 \\ \text { M } & 2.50 & 0.57,4.44 & 100.00 \% & 0.011 & 95\end{array}$

Figure 5. Forest plot of the sub-group analysis by gender assessing the comet assay on lymphocytes cells among professionals occupationally exposed to antineoplastic drugs (ANDs). F, female, M, male.

\subsection{Sub-Group Analysis by Protective Equipment Used}

The sub-group analysis including studies with a low level of protection (considered as no protection at all or use of only gloves and masks) counted six datasets. The pooled ES was 2.19 [(95\% CI $=0.97-3.42), p$-value $<0.001]$ based on 361 subjects (Figure 6a). High statistical heterogeneity was found $\left(\mathrm{Chi}^{2}=93.63, \mathrm{df}=5, \mathrm{I}^{2}=94.66, p\right.$-value $\left.<0.001\right)$. No publication bias was found by visual assessment of the funnel plot, which was confirmed by Egger's linear regression test (intercept 6.52, $\mathrm{t}=1.30, p$-value $=0.265$ ). The sub-group analysis considering a high level of protection (at least gloves, masks, and air-flow cabinet), included six studies, and the pooled ES was 2.13 [ $(95 \% \mathrm{CI}=0.85-3.40)$, $p$-value $=0.001]$ based on 429 subjects (Figure 6b). In this sub-group analysis, Kopjar 2009 was excluded because the exposed group was composed of nurses who used heterogeneous protective equipment (among the 50 subjects, only 3 of them used gloves, masks, and air-flow cabinet, but the results were only shown as aggregated data). High statistical heterogeneity was found $\left(\mathrm{Chi}^{2}=128.11, \mathrm{df}=5\right.$, $\mathrm{I}^{2}=96.10, p$-value $\left.<0.001\right)$. Potential publication bias was found by visual assessment of the funnel plot, and confirmed by Egger's linear regression test (intercept 6.97, $t=2.47, p$-value $=0.069$ ). However, after the trim and fill method was applied, the estimated ES did not change.

$\begin{array}{rccccc} & \text { ES } & \mathbf{9 5 \%} \mathbf{C l} & \text { W } & \text { Sig. } \\ \text { Rekhadevi 2007/Female } & 4.14 & 3.51,4.78 & 17.02 \% & 0.000 & 120 \\ \text { Hongping (a) 2005 } & 2.91 & 2.04,3.78 & 16.37 \% & 0.000 & 42 \\ \text { Hongping (a) } 2006 & 2.50 & 1.54,3.45 & 16.09 \% & 0.000 & 30 \\ \text { Yoshida 2006/Female } & 1.56 & 0.83,2.30 & 16.75 \% & 0.000 & 37 \\ \text { Kopjar (c) 2001 } & 1.42 & 0.52,2.32 & 16.26 \% & 0.002 & 28 \\ \text { Villarini (a) 2011 } & 0.66 & 0.27,1.06 & 17.51 \% & 0.001 & 104 \\ \text { Overall (random-effects model) } & 2.19 & 0.97,3.42 & 100.00 \% & 0.000 & 361\end{array}$

(a)

Figure 6. Cont. 


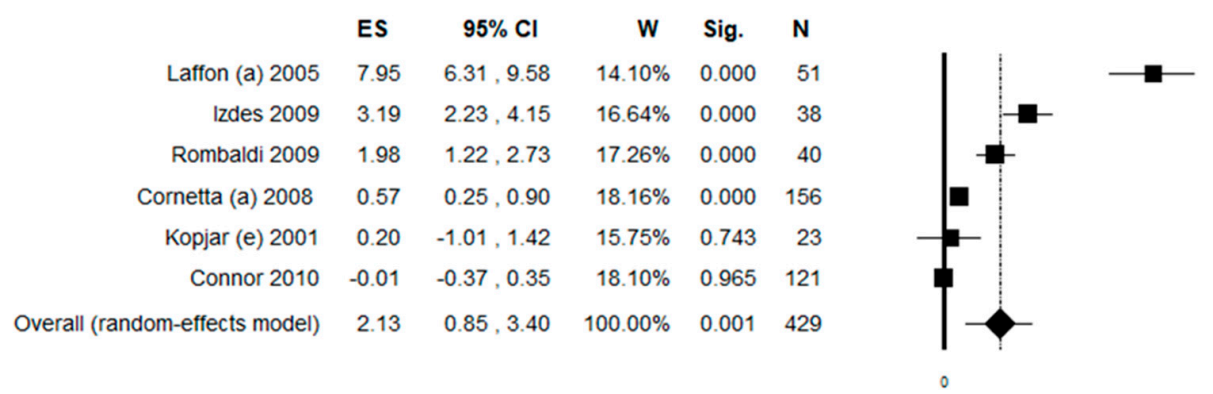

(b)

Figure 6. Forest plot of the sub-group analysis by protective equipment used assessing the comet assay on lymphocytes cells among professionals occupationally exposed to antineoplastic drugs (ANDs). (a) Without any equipment or at least gloves and masks; (b) using at least gloves, masks, and air-flow cabinet.

\subsection{Sub-Group Analysis by Work Task}

In the sub-group analysis by work task, we included only studies that considered, as controls, health professionals not exposed to ANDs different from nurses. In this analysis, 13 datasets were included for females and the pooled ES was 1.24 [ (95\% CI $=0.58-1.89), p$-value $<0.001]$ based on 924 subjects (Figure 7). High statistical heterogeneity was found $\left(\mathrm{Chi}^{2}=217.72, \mathrm{df}=12, \mathrm{I}^{2}=94.49\right.$, $p$-value $<0.001)$. No publication bias was found by visual assessment of the funnel plot and confirmed by Egger's linear regression test (Intercept 4.68, $t=1.33, p$-value $=0.209$ ).

$\begin{array}{rlcccr} & \text { ES } & \mathbf{9 5} \% \mathbf{C l} & \mathbf{W} & \text { Sig. } & \text { N } \\ \text { Rekhadevi 2007/Female } & 4.14 & 3.51,4.78 & 7.73 \% & 0.000 & 120 \\ \text { Izdes 2009 } & 3.19 & 2.23,4.15 & 7.08 \% & 0.000 & 38 \\ \text { kopjar 2009/Female } & 2.45 & 1.93,2.97 & 7.92 \% & 0.000 & 100 \\ \text { Rombaldi 2009 } & 1.98 & 1.22,2.73 & 7.51 \% & 0.000 & 40 \\ \text { Maluf 2000 } & 1.58 & 0.66,2.49 & 7.17 \% & 0.001 & 24 \\ \text { Kopjar (a) 2001 } & 1.32 & 0.76,1.88 & 7.85 \% & 0.000 & 70 \\ \text { Villarini (a) 2011 } & 0.66 & 0.27,1.06 & 8.09 \% & 0.001 & 104 \\ \text { Cornetta (a) 2008 } & 0.57 & 0.25,0.90 & 8.17 \% & 0.000 & 156 \\ \text { Ursini (a) 2006 } & 0.56 & -0.39,1.52 & 7.08 \% & 0.249 & 35 \\ \text { Ladeira 2015 } & 0.31 & -0.10,0.72 & 8.07 \% & 0.135 & 92 \\ \text { Ursini (b) 2006 } & -0.07 & -0.74,0.60 & 7.67 \% & 0.834 & 42 \\ \text { Ursini (c) 2006 } & -0.17 & -0.83,0.48 & 7.70 \% & 0.601 & 43 \\ \text { Cavallo (a) 2009 } & -0.18 & -0.69,0.32 & 7.94 \% & 0.476 & 60 \\ \text { Overall (random-effects model) } & 1.24 & 0.58,1.89 & 100.00 \% & 0.000 & 924\end{array}$

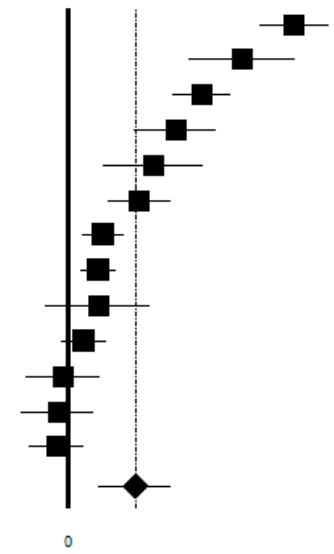

Figure 7. Forest plot of the sub-group analysis by work task (including only studies where controls were other health professionals different from nurses) assessing the comet assay on lymphocytes cells among professionals occupationally exposed to antineoplastic drugs (ANDs).

\subsection{Sub-Group Analysis by Continent}

In the sub-group analysis by continent, the number of datasets of studies conducted in Europe was 11 and the pooled ES was 0.92 [ $(95 \% \mathrm{CI}=0.16-1.68), p$-value $=0.018$ ] based on 890 subjects (Figure 8). High statistical heterogeneity was found $\left(\mathrm{Chi}^{2}=248.68, \mathrm{df}=10, \mathrm{I}^{2}=95.98, p\right.$-value $\left.<0.001\right)$. The number of datasets of studies conducted in Asia was seven and the pooled ES was 1.85 [(95\% CI $=0.32-3.38)$, $p$-value $<0.001$ ] based on 494 subjects (Figure 8 ). High statistical heterogeneity was found $\left(\mathrm{Chi}^{2}=262.49\right.$, $\mathrm{df}=10, \mathrm{I}^{2}=97.42, p$-value $\left.<0.001\right)$. The number of datasets of studies conducted in Americas was three and the pooled ES was 1.15 [ $(95 \% \mathrm{CI}=-0.27-2.56), p$-value $<0.001]$ based on 185 subjects (Figure 8$)$. High statistical heterogeneity was found $\left(\mathrm{Chi}^{2}=27.64, \mathrm{df}=2, \mathrm{I}^{2}=92.76, p\right.$-value $\left.<0.001\right)$. Potential publication bias was found by visual assessment of the funnel plot and confirmed by Egger's linear 
regression test (intercept $8.92, \mathrm{t}=3.54, p$-value $=0.002$ ). However, after applying the trim and fill method, the estimated ES did not change.

\begin{tabular}{rrrrrr} 
& ES & $95 \% \mathrm{Cl}$ & $\mathbf{W}$ & \multicolumn{1}{l}{ Sig. } & \multicolumn{1}{c}{$\mathbf{N}$} \\
Rombaldi 2009 & 1.98 & $1.22,2.73$ & $32.94 \%$ & 0.000 & \multicolumn{1}{c}{40} \\
Maluf 2000 & 1.58 & $0.66,2.49$ & $31.54 \%$ & 0.001 & 24 \\
Connor 2010 & -0.01 & $-0.37,0.35$ & $35.52 \%$ & 0.965 & 121 \\
America & 1.15 & $-0.27,2.56$ & $100.00 \%$ & 0.113 & 185 \\
& & & & & \\
Rekhadevi 2007 & 4.14 & $3.51,4.78$ & $14.43 \%$ & 0.000 & 120 \\
Iddes 2009 & 3.19 & $2.23,4.15$ & $13.98 \%$ & 0.000 & 38 \\
Hongping (a) 2005 & 2.91 & $2.04,3.78$ & $14.12 \%$ & 0.000 & 42 \\
Hongping (a) 2006 & 2.50 & $1.54,3.45$ & $13.99 \%$ & 0.000 & 30 \\
Yoshida 2006 & 1.56 & $0.83,2.30$ & $14.31 \%$ & 0.000 & 37 \\
Sasaki 2008 & 0.47 & $0.13,0.81$ & $14.69 \%$ & 0.007 & 167 \\
Undeger 1999 & -1.71 & $-2.30,-1.12$ & $14.48 \%$ & 0.000 & 60 \\
Asia & 1.85 & $0.32,3.38$ & $100.00 \%$ & 0.018 & 494 \\
& & & & & \\
Laffon (a) 2005 & 7.95 & $6.31,9.58$ & $6.76 \%$ & 0.000 & 51 \\
kopjar 2009 & 2.45 & $1.93,2.97$ & $9.39 \%$ & 0.000 & 100 \\
Kopjar (a) 2001 & 1.32 & $0.76,1.88$ & $9.32 \%$ & 0.000 & 70 \\
Villarini (a) 2011 & 0.66 & $0.27,1.06$ & $9.56 \%$ & 0.001 & 104 \\
Cornetta (a) 2008 & 0.57 & $0.25,0.90$ & $9.65 \%$ & 0.000 & 156 \\
Ursini (a) 2006 & 0.56 & $-0.39,1.52$ & $8.51 \%$ & 0.249 & 35 \\
Ladeira 2015 & 0.31 & $-0.10,0.72$ & $9.54 \%$ & 0.135 & 92 \\
Ursini (b) 2006 & -0.07 & $-0.74,0.60$ & $9.12 \%$ & 0.834 & 42 \\
Ursini (c) 2006 & -0.17 & $-0.83,0.48$ & $9.16 \%$ & 0.601 & 43 \\
Cavallo (a) 2009 & -0.18 & $-0.69,0.32$ & $9.41 \%$ & 0.476 & 60 \\
Buschini 2013 & -1.33 & $-1.70,-0.96$ & $9.59 \%$ & 0.000 & 137 \\
Europe & 0.92 & $0.16,1.68$ & $100.00 \%$ & 0.018 & 890 \\
& & & & &
\end{tabular}

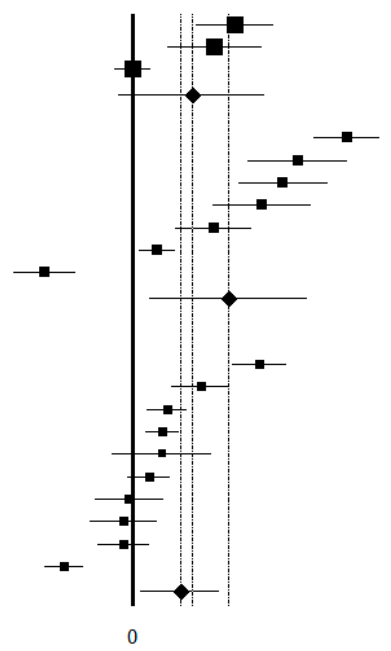

Figure 8. Forest plot of sub-group analysis by continent, assessing the comet assay on lymphocytes cells among professionals occupationally exposed to antineoplastic drugs (ANDs).

\subsection{Cumulative Analysis}

In order to estimate the evolution of the ES across the publications, a cumulative analysis was performed. The cumulative analysis by the year of publication showed a direct association between occupational exposure to ANDs and comet assay, and the results remained stable until the 2008. In 2009, the results started to lose significance, with a large 95\% CI (Figure 9a). Referring to the cumulative analysis for sample size, datasets with small sample showed a larger 95\% CI. On the contrary, the higher the sample size, the more stable the result (Figure 9b).

$\begin{array}{rccccc} & \text { ES } & 95 \% \text { Cl } & \text { W } & \text { Sig. } & \text { N } \\ \text { Ladeira 2015 } & 0.31 & -0.10,0.72 & 4.98 \% & 0.135 & 92 \\ \text { Buschini 2013 } & -0.51 & -2.13,1.10 & 0.00 \% & 0.534 & 229 \\ \text { Villarini (a) 2011 } & -0.12 & -1.36,1.12 & 0.00 \% & 0.849 & 333 \\ \text { Connor 2010 } & -0.09 & -0.96,0.77 & 0.00 \% & 0.832 & 454 \\ \text { Cavallo (a) 2009 } & -0.11 & -0.81,0.59 & 0.00 \% & 0.757 & 514 \\ \text { Izdes 2009 } & 0.37 & -0.47,1.20 & 0.00 \% & 0.388 & 552 \\ \text { Rombaldi 2009 } & 0.59 & -0.24,1.42 & 0.00 \% & 0.161 & 592 \\ \text { kopjar 2009 } & 0.84 & -0.06,1.74 & 0.00 \% & 0.067 & 692 \\ \text { Cornetta (a) 2008 } & 0.80 & 0.05,1.56 & 0.00 \% & 0.038 & 848 \\ \text { Sasaki 2008 } & 0.76 & 0.10,1.41 & 0.00 \% & 0.023 & 1015 \\ \text { Rekhadevi 2007 } & 1.08 & 0.30,1.85 & 0.00 \% & 0.006 & 1135 \\ \text { Hongping (a) 2006 } & 1.19 & 0.44,1.94 & 0.00 \% & 0.002 & 1165 \\ \text { Ursini (a) 2006 } & 1.14 & 0.43,1.86 & 0.00 \% & 0.002 & 1200 \\ \text { Ursini (b) 2006 } & 1.05 & 0.38,1.73 & 0.00 \% & 0.002 & 1242 \\ \text { Ursini (c) 2006 } & 0.97 & 0.33,1.62 & 0.00 \% & 0.003 & 1285 \\ \text { Yoshida 2006 } & 1.01 & 0.39,1.63 & 0.00 \% & 0.001 & 1322 \\ \text { Hongping (a) 2005 } & 1.12 & 0.50,1.73 & 0.00 \% & 0.000 & 1364 \\ \text { Laffon (a) 2005 } & 1.42 & 0.76,2.07 & 0.00 \% & 0.000 & 1415 \\ \text { Kopjar (a) 2001 } & 1.41 & 0.78,2.03 & 0.00 \% & 0.000 & 1485 \\ \text { Maluf 2000 } & 1.42 & 0.81,2.02 & 0.00 \% & 0.000 & 1509 \\ \text { Undeger 1999 } & 1.27 & 0.66,1.88 & 0.00 \% & 0.000 & 1569\end{array}$

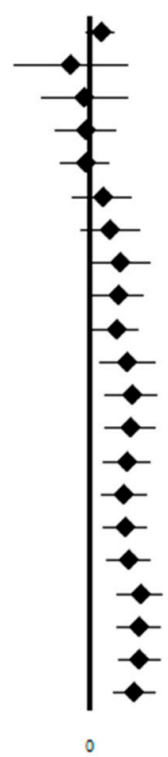

(a)

Figure 9. Cont. 


$\begin{array}{rccc} & \text { ES } & 95 \% \text { Cl } & \text { W } \\ \text { Sasaki 2008 } & 0.47 & 0.13,0.81 & 0.00 \% \\ \text { Cornetta (a) 2008 } & 0.52 & 0.29,0.76 & 0.00 \% \\ \text { Buschini 2013 } & -0.09 & -1.26,1.07 & 0.00 \% \\ \text { Connor 2010 } & -0.07 & -0.91,0.76 & 0.00 \% \\ \text { Rekhadevi 2007 } & 0.75 & -0.52,2.01 & 0.00 \% \\ \text { Villarini (a) 2011 } & 0.73 & -0.32,1.78 & 0.00 \% \\ \text { kopjar 2009 } & 0.97 & -0.05,2.00 & 0.00 \% \\ \text { Ladeira 2015 } & 0.89 & -0.01,1.78 & 0.00 \% \\ \text { Kopjar (a) 2001 } & 0.94 & 0.11,1.76 & 0.00 \% \\ \text { Cavallo (a) 2009 } & 0.82 & 0.06,1.58 & 0.00 \% \\ \text { Undeger 1999 } & 0.60 & -0.16,1.36 & 0.00 \% \\ \text { Laffon (a) 2005 } & 1.08 & 0.26,1.89 & 0.00 \% \\ \text { Ursini (c) 2006 } & 0.98 & 0.21,1.74 & 0.00 \% \\ \text { Hongping (a) 2005 } & 1.11 & 0.36,1.87 & 0.00 \% \\ \text { Ursini (b) 2006 } & 1.03 & 0.31,1.75 & 0.00 \% \\ \text { Rombaldi 2009 } & 1.09 & 0.39,1.79 & 0.00 \% \\ \text { Izdes 2009 } & 1.21 & 0.52,1.90 & 0.00 \% \\ \text { Yoshida 2006 } & 1.23 & 0.56,1.89 & 0.00 \% \\ \text { Ursini (a) 2006 } & 1.19 & 0.55,1.84 & 0.00 \% \\ \text { Hongping (a) 2006 } & 1.26 & 0.62,1.89 & 0.00 \% \\ \text { Maluf 2000 } & 1.27 & 0.66,1.88 & 0.00 \%\end{array}$

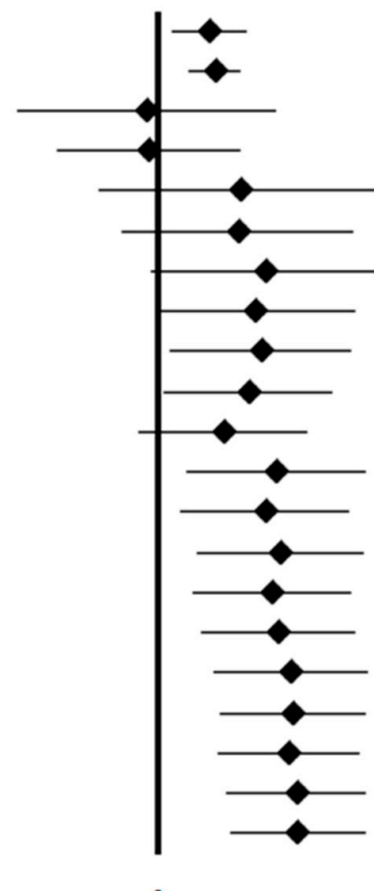

(b)

Figure 9. Cumulative analysis by (a) year of publication (descending order) and (b) effect size (ES) (from the smallest to the biggest) assessing the comet assay on lymphocytes cells among professionals occupationally exposed to antineoplastic drugs (ANDs).

\subsection{Moderator Analysis}

Considering the year of publication as covariate in the moderator analysis, the results changed significantly between the three groups of years. Particularly, the early studies (before 2004) did not find a statistically significant association between occupational exposure to ANDs and comet assay. Considering the time period 2005-2010, a higher number of studies were conducted, increasing the sample size $(n=1082)$ and stabilizing the results. However, considering only studies conducted after 2010, only three datasets were included, reducing the sample size $(n=333)$ and increasing the uncertainty of the results, confirmed by the large $95 \% \mathrm{CI}$ (Figure 10a). The results of the meta-regression analysis by duration of the exposure showed a linear association; a higher duration of exposure was associated with a higher ES (Figure 10b). The association was less clear when the age of exposed groups was considered (Figure 10c). 


\begin{tabular}{rrrrrr} 
& ES & \multicolumn{1}{c}{$95 \%$ Cl } & W & Sig. & \multicolumn{1}{c}{ N } \\
Laffon (a) 2005 & 7.95 & $6.31,9.58$ & $5.30 \%$ & 0.000 & 51 \\
Rekhadevi 2007 & 4.14 & $3.51,4.78$ & $6.82 \%$ & 0.000 & 120 \\
Izdes 2009 & 3.19 & $2.23,4.15$ & $6.41 \%$ & 0.000 & 38 \\
Hongping (a) 2005 & 2.91 & $2.04,3.78$ & $6.54 \%$ & 0.000 & 42 \\
Hongping (a) 2006 & 2.50 & $1.54,3.45$ & $6.41 \%$ & 0.000 & 30 \\
kopjar 2009 & 2.45 & $1.93,2.97$ & $6.94 \%$ & 0.000 & 100 \\
Rombaldi 2009 & 1.98 & $1.22,2.73$ & $6.68 \%$ & 0.000 & 40 \\
Yoshida 2006 & 1.56 & $0.83,2.30$ & $6.71 \%$ & 0.000 & 37 \\
Cornetta (a) 2008 & 0.57 & $0.25,0.90$ & $7.09 \%$ & 0.000 & 156 \\
Ursini (a) 2006 & 0.56 & $-0.39,1.52$ & $6.41 \%$ & 0.249 & 35 \\
Sasaki 2008 & 0.47 & $0.13,0.81$ & $7.08 \%$ & 0.007 & 167 \\
Connor 2010 & -0.01 & $-0.37,0.35$ & $7.07 \%$ & 0.965 & 121 \\
Ursini (b) 2006 & -0.07 & $-0.74,0.60$ & $6.79 \%$ & 0.834 & 42 \\
Ursini (c) 2006 & -0.17 & $-0.83,0.48$ & $6.81 \%$ & 0.601 & 43 \\
Cavallo (a) 2009 & -0.18 & $-0.69,0.32$ & $6.95 \%$ & 0.476 & 60 \\
2005-2010 & 1.75 & $1.01,2.48$ & $100.00 \%$ & 0.000 & 1082 \\
& & & & & \\
Maluf 2000 & 1.58 & $0.66,2.49$ & $32.55 \%$ & 0.001 & 24 \\
Kopjar (a) 2001 & 1.32 & $0.76,1.88$ & $33.76 \%$ & 0.000 & 70 \\
Undeger 1999 & -1.71 & $-2.30,-1.12$ & $33.68 \%$ & 0.000 & 60 \\
$<=2004$ & 0.38 & $-1.80,2.57$ & $100.00 \%$ & 0.729 & 154 \\
& & & & & \\
Villarini (a) 2011 & 0.66 & $0.27,1.06$ & $33.32 \%$ & 0.001 & 104 \\
Ladeira 2015 & 0.31 & $-0.10,0.72$ & $33.23 \%$ & 0.135 & 92 \\
Buschini 2013 & -1.33 & $-1.70,-0.96$ & $33.45 \%$ & 0.000 & 137 \\
$>2010$ & -0.12 & $-1.36,1.12$ & $100.00 \%$ & 0.849 & 333
\end{tabular}

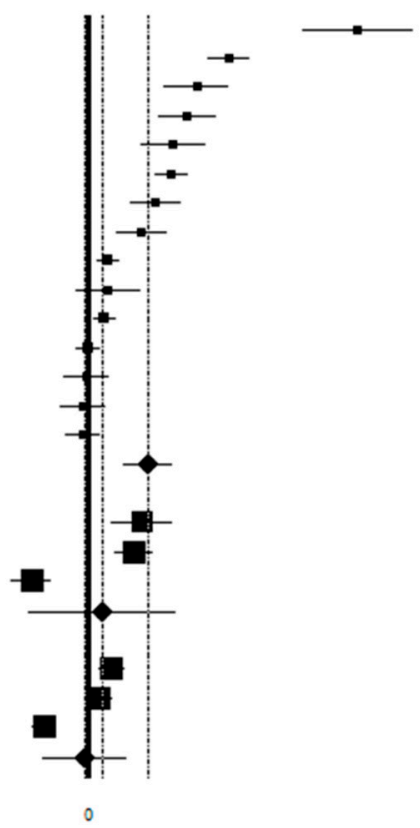

(a)

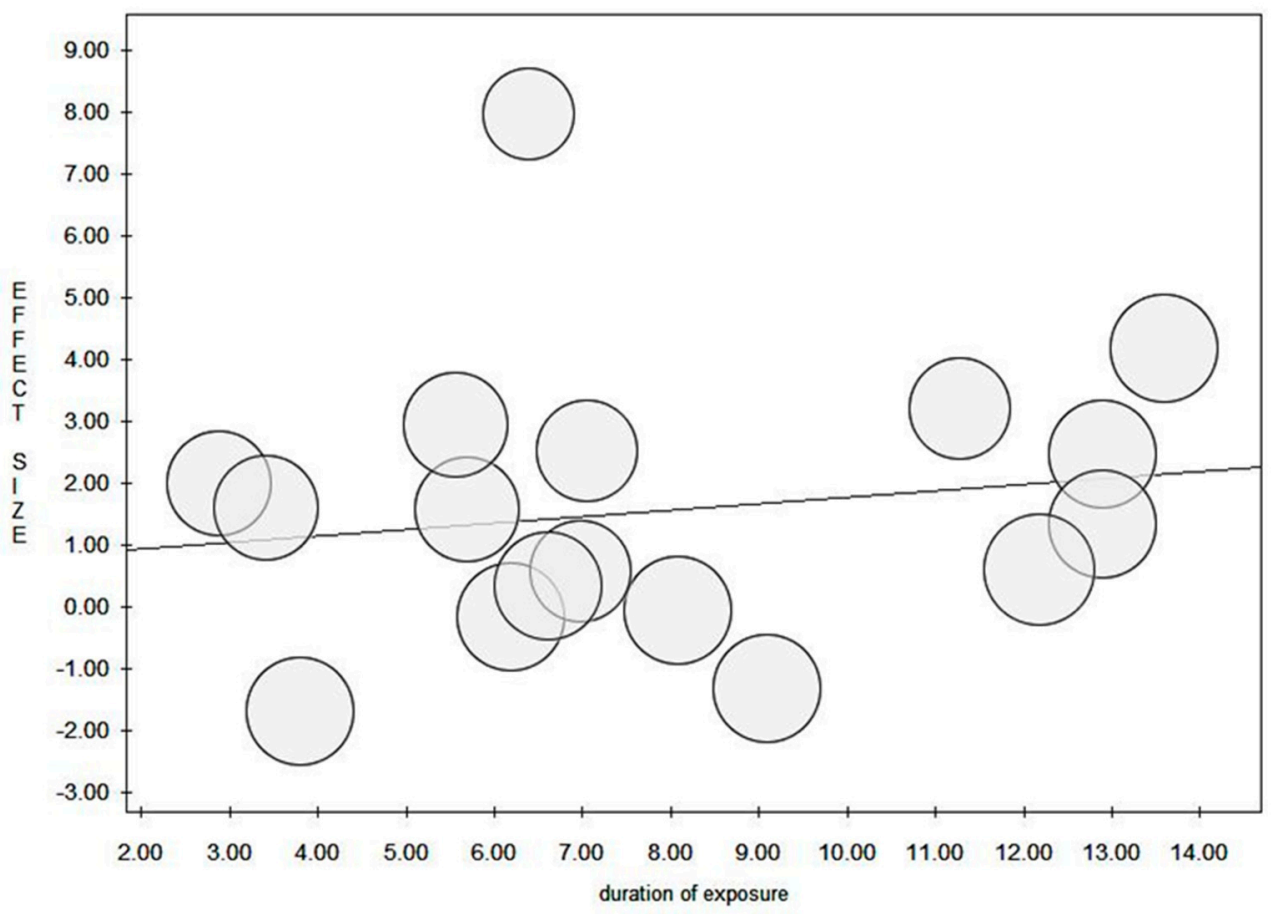

(b)

Figure 10. Cont. 


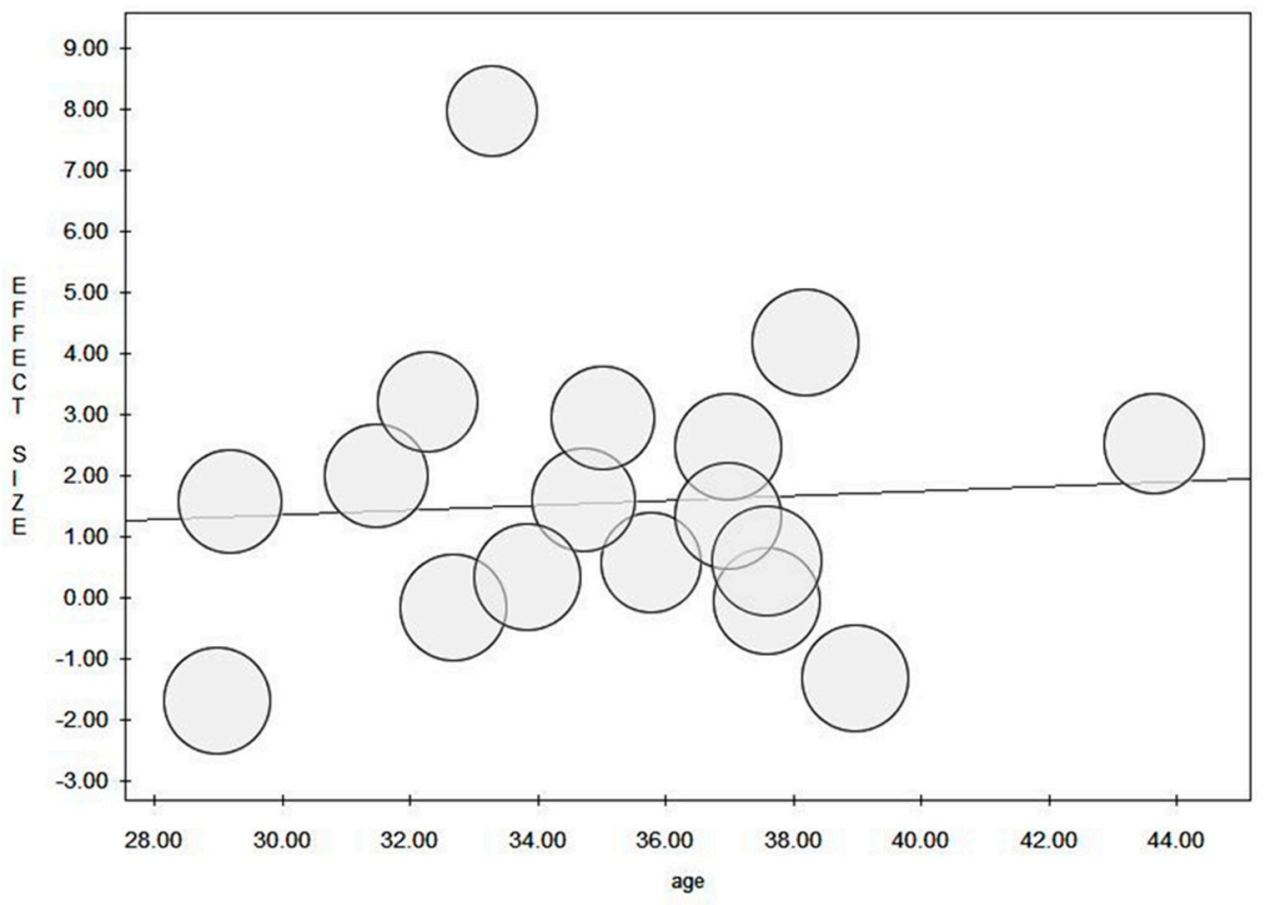

(c)

Figure 10. (a) Moderator analysis by year of publication (the first group consisted of studies until 2004, the second between 2005 and 2010, and the third after 2010 until today); (b) meta-regression analysis by duration of exposure; and (c) meta-regression analysis by mean age of exposed groups, assessing the comet assay on lymphocytes cells among professionals occupationally exposed to antineoplastic drugs (ANDs).

\section{Discussion}

The current systematic review with meta-analysis-which included 20 studies in the qualitative evaluation and 19 studies in the quantitative analysis-provided data on the association between occupational exposure to ANDs and the extent of primary DNA damage, as evaluated by the comet assay. As some studies separately reported data for gender, work task, and protective equipment, and a total of 21 datasets were considered. The pooled ES based on 1569 subjects indicated a significantly higher extent of primary DNA damage in health professionals exposed to ANDs compared with controls, with an ES of 1.27 [(95\% CI $=0.66-1.88), p$-value $<0.001]$. The ES did not change significantly when, in the sub-group analysis, other health professionals different from nurses were considered as controls, even if it was slightly lower [1.24 (95\% CI $=0.58-1.89), p$-value $<0.001]$.

In order to deeply understand the strength of the association between occupational exposure and primary DNA damage, a sub-group analysis by protective equipment was conducted. When studies that assessed the damage among health professionals who did not use any protective equipment or only masks and gloves were considered, the pooled ES was higher compared with the studies where health professionals used a combination of personal and environmental protective equipment (at least gloves, masks, and airflow cabinet). The importance of protective equipment could explain the differences obtained in the sub-group analysis by continent. Particularly, studies conducted in Asia showed the highest pooled ES compared with studies conducted in the Americas and even more in Europe. As suggested by Hon et al., different standard procedures, protocols, level of education, and legislations across the globe might influence the level of occupational risk, and consequently the conclusions drawn from one country might not be fully applicable to another [50]. Furthermore, in the cumulative analysis by year of publication, the results remained stable until 2008, while from 2009, they started to lose significance, with a large $95 \%$ CI. It should be noticed that a large number of guidelines on ANDs' manipulation, aimed to reduce the occupational risk, were published between 2000 and 
2009 [27-29,51]. Probably, the reinforcement of protective occupational strategies, the higher attention reserved to the occupational health risk, and particularly the awareness of ANDs dangerousness even at low doses might have played an important role in reducing the occupational exposure risk. However, guidelines alone are not enough, because health professionals need to be empowered with regards to potential and accidental contaminations through tailored training and communication [52]. As a matter of fact, even if health professionals are exposed to a low or very low dose of ANDs, the exposure is nearly daily and prolonged at times. According to our results, the duration of exposure was an important moderator of the association between ANDs exposure and primary DNA damage. This result is in contrast with the data obtained by Mader et al.- the only study that stratified the results according to the duration of exposure (expressed in years) [49]. Considering the sub-group analysis by gender, the pooled ES was marginally higher among men compared with women. However, these data should be read with caution. Firstly, the total datasets assessing primary DNA damage among men were only 5, while 10 datasets performed the comet assay among women; secondly, the men's sample size was 10 times lower compared with that of the women; thirdly, the 95\% CI was wider among men. An important aspect should be considered before the generalization of these results, that is, even though several sub-group analyses were conducted, the value of heterogeneity remained stably high. Indeed, although a sensitivity analysis including only datasets with a high quality score (QS $\geq 16$ ) was conducted, the $\mathrm{I}^{2}$ remained around $95 \%$. Only in one case was the heterogeneity below $40 \%$, when datasets performing the comet assay on buccal cells were considered. However, only four datasets provided this data, resulting in a small sample size ( $n=180$ subjects), wide $95 \% \mathrm{CI}$, and loss of statistical significance. The high value of heterogeneity found could be explained considering the methodological variation of the comet assay used in the considered primary studies. Indeed, primary papers expressed the results of the comet assay using different types of unit of measures. Moreover, an $\mathrm{I}^{2}$ value higher than $90 \%$ means that heterogeneity is directly the result of heterogeneity among studies, rather than sampling errors [53]. Even if the pooled ES was estimated by Cohen's d, allowing comparability, this underlying heterogeneity might have affected the assessment of the $\mathrm{I}^{2}$. Another potential explanation of heterogeneity could be the different type of ANDs used or the type of exposure (not only duration or work task, but also doses and therapeutic scheme used). Moreover, even if the primary studies tried to match exposed and control subjects for several confounders, only a few studies perfectly matched for the most important elements, such as smoking, alcohol, and nutritional intake. Nevertheless, there are several strengths in this study: first, this is an extensive systematic review conducted according the PRISMA guidelines, which allowed retrieving a large number of studies; second, the pooled ES was based on 1569 subjects; and third, several sub-group analyses as well as sensitivity and moderator analyses were conducted. Particularly, the ES was estimated in both women and men, considering the work task and the protective equipment used. Moreover, in the meta-regression, the duration of exposure was considered as moderator. Furthermore, whenever we found potential publication bias, a trim and fill method was applied. Even if high heterogeneity was found, a random effect model was considered. Lastly, the pooled ES was estimated both in lymphocytes and buccal cells.

\section{Conclusions}

To conclude, the results of this systematic review with meta-analysis clearly show a statistically significant association between occupational exposure to ANDs and the extent of primary DNA damage. The comet assay method is able to identify reversible DNA damage; in this perspective, the identified damage is still potentially repairable. However, a prolonged exposure, a concomitant action of other risk factors, and the senescence of DNA repair systems might induce a risk of neoplasms or other side effects (such as, for instance, a teratogenic action). Having said that, health professionals should be made aware of this potential occupational risk.

Author Contributions: V.G. and M.M. conceived and designed the study. V.G., C.F., and T.S. searched and abstracted the literature, and extracted meta-data from the included manuscripts. V.G. and D.N. performed analysis and interpretation of data. M.M. assisted with interpretation of the data and acted as the third (mediating) 
reviewer. M.M. and M.V. provided critical revision of drafts. V.G. and D.N. wrote the first draft of the manuscript and revised the content based on feedback. All the authors contributed to the interpretation of the results and performed a critical revision of the manuscript. All authors approved the final version before submission. All authors have read and agreed to the published version of the manuscript.

Funding: This research received no external funding.

Acknowledgments: Authors would like to thank Valeria Parisi for assisting in figure preparation, and Dr. Federica Balzarini and Dr. Giulia Dallagiacoma for English revision.

Conflicts of Interest: The authors declare no conflict of interest.

\section{References}

1. Krumbhaar, E.B.; Krumbhaar, H.D. The Blood and Bone Marrow in Yelloe Cross Gas (Mustard Gas) Poisoning: Changes produced in the Bone Marrow of Fatal Cases. J. Med. Res. 1919, 40, 497-508.

2. Alexander, S.F. Medical report on the Bari Harbor mustard casualties. Mil. Surg. 1947, 101, 1-17.

3. Gilman, A.; Philips, F.S. The biological actions and therapeutic applications of the B-chloroethyl amines and sulfides. Science 1946, 103, 409-415. [CrossRef]

4. Gianfredi, V.; Acito, M.; Salvatori, T.; Villarini, M.; Moretti, M. Use of Micronucleus Assays to Measure DNA Damage Caused by Cytostatic/Antineoplastic Drugs. In Issues in Toxicology; Fenech, M., Knasmuller, S., Eds.; Royal Society of Chemistry: London, UK, 2019; pp. 601-617.

5. IARC. A Review of Human Carcinogens. Part A: Pharmaceuticals. IARC Monographs on the Evaluation of Carcinogenic Risks to Humans; International Agency for Research on Cancer: Lyon, France, 2011; Volume 100.

6. Gianfredi, V.; Salvatori, T.; Nucci, D.; Villarini, M.; Moretti, M. Genotoxic risk in nurses handling antiblastic drugs: Systematic review of literature and meta-analysis. Recenti Prog. Med. 2017, 108, 511-520. [PubMed]

7. Villarini, M.; Gianfredi, V.; Levorato, S.; Vannini, S.; Salvatori, T.; Moretti, M. Occupational exposure to cytostatic/antineoplastic drugs and cytogenetic damage measured using the lymphocyte cytokinesis-block micronucleus assay: A systematic review of the literature and meta-analysis. Mutat. Res. 2016, 770 Pt A, 35-45. [CrossRef] [PubMed]

8. Dranitsaris, G.; Johnston, M.; Poirier, S.; Schueller, T.; Milliken, D.; Green, E.; Zanke, B. Are health care providers who work with cancer drugs at an increased risk for toxic events? A systematic review and meta-analysis of the literature. J. Oncol. Pharm. Pract. 2005, 11, 69-78. [CrossRef] [PubMed]

9. McDiarmid, M.; Egan, T. Acute occupational exposure to antineoplastic agents. J. Occup. Med. 1988, 30, 984-987. [CrossRef]

10. Valanis, B.; Vollmer, W.M.; Steele, P. Occupational exposure to antineoplastic agents: Self-reported miscarriages and stillbirths among nurses and pharmacists. J. Occup. Environ. Med. 1999, 41, 632-638. [CrossRef]

11. Honardoost, M.; Rajabpour, A.; Vakili, L. Molecular epidemiology; New but impressive. Med. J. Islamic Repub. Iran 2018, 32, 53. [CrossRef]

12. Ostling, O.; Johanson, K.J. Microelectrophoretic study of radiation-induced DNA damages in individual mammalian cells. Biochem. Biophys. Res. Commun. 1984, 123, 291-298. [CrossRef]

13. Singh, N.P.; McCoy, M.T.; Tice, R.R.; Schneider, E.L. A simple technique for quantitation of low levels of DNA damage in individual cells. Exp. Cell Res. 1988, 175, 184-191. [CrossRef]

14. Collins, A.R.; Oscoz, A.A.; Brunborg, G.; Gaivao, I.; Giovannelli, L.; Kruszewski, M.; Smith, C.C.; Stetina, R. The comet assay: Topical issues. Mutagenesis 2008, 23, 143-151. [CrossRef] [PubMed]

15. Singh, N.P.; Tice, R.R.; Stephens, R.E.; Schneider, E.L. A microgel electrophoresis technique for the direct quantitation of DNA damage and repair in individual fibroblasts cultured on microscope slides. Mutat. Res. 1991, 252, 289-296. [CrossRef]

16. Valverde, M.; Rojas, E. Environmental and occupational biomonitoring using the Comet assay. Mutat. Res. 2009, 681, 93-109. [CrossRef]

17. Collins, A.; Anderson, D.; Coskun, E.; Dhawan, A.; Dusinska, M.; Koppen, G.; Kruszewski, M.; Moretti, M.; Rojas, E.; Speit, G.; et al. Launch of the ComNet (comet network) project on the comet assay in human population studies during the International Comet Assay Workshop meeting in Kusadasi, Turkey (13-16 September 2011). Mutagenesis 2012, 27, 385-386. [CrossRef] 
18. Collins, A.; Koppen, G.; Valdiglesias, V.; Dusinska, M.; Kruszewski, M.; Moller, P.; Rojas, E.; Dhawan, A.; Benzie, I.; Coskun, E.; et al. The comet assay as a tool for human biomonitoring studies: The ComNet project. Mutat. Res. Rev. Mutat. Res. 2014, 759, 27-39. [CrossRef]

19. Moher, D.; Shamseer, L.; Clarke, M.; Ghersi, D.; Liberati, A.; Petticrew, M.; Shekelle, P.; Stewart, L.A.; Group, P.-P. Preferred reporting items for systematic review and meta-analysis protocols (PRISMA-P) 2015 statement. Syst. Rev. 2015, 4, 1. [CrossRef]

20. Brown, P.; Brunnhuber, K.; Chalkidou, K.; Chalmers, I.; Clarke, M.; Fenton, M.; Forbes, C.; Glanville, J.; Hicks, N.J.; Moody, J.; et al. How to formulate research recommendations. BMJ 2006, 333, 804-806. [CrossRef]

21. Higgins, J.P.T.; Green, S. Cochrane Handbook for Systematic Reviews of Interventions; Version 5.1.0; The Cochrane Collaboration: Melbourne, Australia, 2013.

22. Rhea, M.R. Determining the magnitude of treatment effects in strength training research through the use of the effect size. J. Strength Cond. Res. 2004, 18, 918-920.

23. Egger, M.; Davey Smith, G.; Schneider, M.; Minder, C. Bias in meta-analysis detected by a simple, graphical test. BMJ 1997, 315, 629-634. [CrossRef]

24. Duval, S.; Tweedie, R. A nonparametric "Trim and Fill" method of accounting for Publication Bias in Meta-Analysis. J. Am. Stat. Assoc. 2000, 95, 89-98.

25. Shi, L.; Lin, L. The trim-and-fill method for publication bias: Practical guidelines and recommendations based on a large database of meta-analyses. Medicine 2019, 98, e15987. [CrossRef] [PubMed]

26. Leimu, R.; Koricheva, J. Cumulative meta-analysis: A new tool for detection of temporal trends and publication bias in ecology. Proc. Biol. Sci. 2004, 271, 1961-1966. [CrossRef] [PubMed]

27. NIOSH. NIOSH Alert: Preventing Occupational Exposures to Antineoplastic and Other Hazardous Drugs in Health Care Settings; National Institute for Occupational Safety and Health: Cincinnati, OH, USA, 2004.

28. ASHP. ASHP (American Society of Hospital Pharmacists) guidelines on handling hazardous drugs. Am. J. Hosp. Pharm. 2006, 63, 1172-1193.

29. Schierl, R.; Bohlandt, A.; Nowak, D. Guidance values for surface monitoring of antineoplastic drugs in German pharmacies. Ann. Occup. Hyg. 2009, 53, 703-711.

30. Buschini, A.; Villarini, M.; Feretti, D.; Mussi, F.; Dominici, L.; Zerbini, I.; Moretti, M.; Ceretti, E.; Bonfiglioli, R.; Carrieri, M.; et al. Multicentre study for the evaluation of mutagenic/carcinogenic risk in nurses exposed to antineoplastic drugs: Assessment of DNA damage. Occup. Environ. Med. 2013, 70, 789-794. [CrossRef]

31. Cavallo, D.; Ursini, C.L.; Rondinone, B.; Iavicoli, S. Evaluation of a suitable DNA damage biomarker for human biomonitoring of exposed workers. Environ. Mol. Mutagenes. 2009, 50, 781-790. [CrossRef]

32. Connor, T.H.; DeBord, D.G.; Pretty, J.R.; Oliver, M.S.; Roth, T.S.; Lees, P.S.; Krieg, E.F., Jr.; Rogers, B.; Escalante, C.P.; Toennis, C.A.; et al. Evaluation of antineoplastic drug exposure of health care workers at three university-based US cancer centers. J. Occup. Environ. Med. 2010, 52, 1019-1027. [CrossRef]

33. Cornetta, T.; Padua, L.; Testa, A.; Ievoli, E.; Festa, F.; Tranfo, G.; Baccelliere, L.; Cozzi, R. Molecular biomonitoring of a population of nurses handling antineoplastic drugs. Mutat. Res. 2008, 638, 75-82. [CrossRef]

34. Hongping, D.; Jianlin, L.; Meibian, Z.; Wei, W.; Lifen, J.; Shijie, C.; Wei, Z.; Baohong, W.; Jiliang, H. Detecting the cytogenetic effects in workers occupationally exposed to vincristine with four genetic tests. Mutat. Res. 2006, 599, 152-159. [CrossRef] [PubMed]

35. Hongping, D.; Zhang, M.; He, J.; Wu, W.; Jin, L.; Zheng, W.; Lou, J.; Wang, B. Investigating genetic damage in workers occupationally exposed to methotrexate using three genetic end-points. Mutagenesis 2005, 20, 351-357.

36. Izdes, S.; Sardas, S.; Kadioglu, E.; Kaymak, C.; Ozcagli, E. Assessment of genotoxic damage in nurses occupationally exposed to anaesthetic gases or antineoplastic drugs by the comet assay. J. Occup. Health 2009, 51, 283-286. [CrossRef] [PubMed]

37. Kopjar, N.; Garaj-Vrhovac, V. Application of the alkaline comet assay in human biomonitoring for genotoxicity: A study on Croatian medical personnel handling antineoplastic drugs. Mutagenesis 2001, 16, 71-78. [CrossRef]

38. Kopjar, N.; Garaj-Vrhovac, V.; Kasuba, V.; Rozgaj, R.; Ramic, S.; Pavlica, V.; Zeljezic, D. Assessment of genotoxic risks in Croatian health care workers occupationally exposed to cytotoxic drugs: A multi-biomarker approach. Int. J. Hyg. Environ. Health 2009, 212, 414-431. [CrossRef] [PubMed] 
39. Ladeira, C.; Viegas, S.; Padua, M.; Carolino, E.; Gomes, M.C.; Brito, M. Relation between DNA damage measured by comet assay and OGG1 Ser326Cys polymorphism in antineoplastic drugs biomonitoring. Aims Genet. 2015, 2, 204-218. [CrossRef]

40. Laffon, B.; Teixeira, J.P.; Silva, S.; Loureiro, J.; Torres, J.; Pasaro, E.; Mendez, J.; Mayan, O. Genotoxic effects in a population of nurses handling antineoplastic drugs, and relationship with genetic polymorphisms in DNA repair enzymes. Am. J. Ind. Med. 2005, 48, 128-136. [CrossRef] [PubMed]

41. Maluf, S.W.; Erdtmann, B. Evaluation of occupational genotoxic risk in a Brazilian hospital. Genet. Mol. Biol. 2000, 23, 485-488. [CrossRef]

42. Rekhadevi, P.V.; Sailaja, N.; Chandrasekhar, M.; Mahboob, M.; Rahman, M.F.; Grover, P. Genotoxicity assessment in oncology nurses handling anti-neoplastic drugs. Mutagenesis 2007, 22, 395-401. [CrossRef]

43. Rombaldi, F.; Cassini, C.; Salvador, M.; Saffi, J.; Erdtmann, B. Occupational risk assessment of genotoxicity and oxidative stress in workers handling anti-neoplastic drugs during a working week. Mutagenesis 2009, 24, 143-148. [CrossRef]

44. Sasaki, M.; Dakeishi, M.; Hoshi, S.; Ishii, N.; Murata, K. Assessment of DNA damage in Japanese nurses handling antineoplastic drugs by the comet assay. J. Occup. Health 2008, 50, 7-12. [CrossRef]

45. Undeger, U.; Basaran, N.; Kars, A.; Guc, D. Assessment of DNA damage in nurses handling antineoplastic drugs by the alkaline COMET assay. Mutat. Res. 1999, 439, 277-285. [CrossRef]

46. Ursini, C.L.; Cavallo, D.; Colombi, A.; Giglio, M.; Marinaccio, A.; Iavicoli, S. Evaluation of early DNA damage in healthcare workers handling antineoplastic drugs. Int. Arch. Occup. Environ. Health 2006, 80, 134-140. [CrossRef] [PubMed]

47. Villarini, M.; Dominici, L.; Piccinini, R.; Fatigoni, C.; Ambrogi, M.; Curti, G.; Morucci, P.; Muzi, G.; Monarca, S.; Moretti, M. Assessment of primary, oxidative and excision repaired DNA damage in hospital personnel handling antineoplastic drugs. Mutagenesis 2011, 26, 359-369. [CrossRef] [PubMed]

48. Yoshida, J.; Kosaka, H.; Tomioka, K.; Kumagai, S. Genotoxic risks to nurses from contamination of the work environment with antineoplastic drugs in Japan. J. Occup. Health 2006, 48, 517-522. [CrossRef] [PubMed]

49. Mader, R.M.; Kokalj, A.; Kratochvil, E.; Pilger, A.; Rudiger, H.W. Longitudinal biomonitoring of nurses handling antineoplastic drugs. J. Clin. Nurs. 2009, 18, 263-269. [CrossRef]

50. Hon, C.Y.; Barzan, C.; Astrakianakis, G. Identification of Knowledge Gaps Regarding Healthcare Workers Exposure to Antineoplastic Drugs: Review of Literature, North America versus Europe. Saf. Health Work 2014, 5, 169-174. [CrossRef]

51. OSHA. OSHA Technical Manual. Hospital Investigations: Health Hazard—Section VI. 2000, Occupational Safety and Health Administration; US Department of Labor: Washington, DC, USA, 2000.

52. Gianfredi, V.; Grisci, C.; Nucci, D.; Parisi, V.; Moretti, M. Communication in health. Recenti Prog. Med. 2018, 109, 374-383.

53. Huedo-Medina, T.B.; Sanchez-Meca, J.; Marin-Martinez, F.; Botella, J. Assessing heterogeneity in meta-analysis: Q statistic or I2 index? Psychol. Methods 2006, 11, 193-206. [CrossRef]

(C) 2020 by the authors. Licensee MDPI, Basel, Switzerland. This article is an open access article distributed under the terms and conditions of the Creative Commons Attribution (CC BY) license (http://creativecommons.org/licenses/by/4.0/). 